\title{
Observation of Higgs Boson Decay to Bottom Quarks
}

\author{
A. M. Sirunyan et al. \\ (CMS Collaboration)
}

(Received 24 August 2018; published 17 September 2018)

\begin{abstract}
The observation of the standard model (SM) Higgs boson decay to a pair of bottom quarks is presented. The main contribution to this result is from processes in which Higgs bosons are produced in association with a $W$ or $Z$ boson $(V H)$, and are searched for in final states including 0 , 1, or 2 charged leptons and two identified bottom quark jets. The results from the measurement of these processes in a data sample recorded by the CMS experiment in 2017, comprising $41.3 \mathrm{fb}^{-1}$ of proton-proton collisions at $\sqrt{s}=13 \mathrm{TeV}$, are described. When combined with previous $V H$ measurements using data collected at $\sqrt{s}=7,8$, and $13 \mathrm{TeV}$, an excess of events is observed at $m_{H}=125 \mathrm{GeV}$ with a significance of 4.8 standard deviations, where the expectation for the SM Higgs boson is 4.9. The corresponding measured signal strength is $1.01 \pm 0.22$. The combination of this result with searches by the CMS experiment for $H \rightarrow b \bar{b}$ in other production processes yields an observed (expected) significance of 5.6 (5.5) standard deviations and a signal strength of $1.04 \pm 0.20$.
\end{abstract}

DOI: 10.1103/PhysRevLett.121.121801

Since the discovery of a new boson with a mass near $125 \mathrm{GeV}$ by the ATLAS [1] and CMS [2,3] Collaborations, rapid progress in the understanding of its properties and couplings has revealed that the new particle is compatible with the standard model (SM) Higgs boson $H$ [4-9]. Observation of Higgs boson decays in the $\gamma \gamma, \mathrm{ZZ}, W W$, and $\tau \tau$ modes have been reported [10-20], and all measured properties [21-29] support this hypothesis. Recently, the CMS and ATLAS Collaborations reported observations of the Higgs boson produced in association with a top quark pair that are compatible with the SM prediction, representing the first direct measurements of the Higgs boson coupling to quarks $[30,31]$.

The decay $H \rightarrow b \bar{b}$, with a predicted branching fraction of about 58\% [32] for a Higgs boson mass of $m_{H}=125 \mathrm{GeV}$, is the most prevalent decay mode but has not yet been established unequivocally. A precise measurement of the rate for this process directly probes the Yukawa coupling of the Higgs boson to a down-type quark, and provides a necessary test of the hypothesis that the Higgs field is the source of mass generation in the charged fermion sector of the SM $[33,34]$. At both the LHC and the Tevatron the most sensitive production process in the search for $H \rightarrow b \bar{b}$ decays is when the Higgs boson is produced in association with a vector boson $(\mathrm{VH})$. The

\footnotetext{
*Full author list given at the end of the article.

Published by the American Physical Society under the terms of the Creative Commons Attribution 4.0 International license. Further distribution of this work must maintain attribution to the author(s) and the published article's title, journal citation, and DOI. Funded by SCOAP.
}

CDF and D0 Collaborations at the Tevatron reported an excess of events in this channel with a significance of 2.8 standard deviations $(\sigma)$ at a mass of $m_{H}=125 \mathrm{GeV}$ [35]. Last year, the ATLAS and CMS Collaborations reported evidence for the $V H, H \rightarrow b \bar{b}$ process at a mass of $m_{H}=125 \mathrm{GeV}$ corresponding to observed (expected) significances of $3.6(4.0) \sigma$ and $3.8(3.8) \sigma$, respectively, combining data collected during run 1 at $\sqrt{s}=7$ and $8 \mathrm{TeV}$, and run 2 at $13 \mathrm{TeV}$ [36,37]. Searches for the $H \rightarrow b \bar{b}$ decay in other production processes, with less sensitivity than $V H$, have also been reported at the LHC [38-44].

In this Letter we present the observation of the Higgs boson decay to bottom quarks. The measurement described here examines the $V H$ production process, where the Higgs boson is produced in association with a $W$ or $Z$ boson and decays into $b \bar{b}$. The data comprise proton-proton $(p p)$ collisions recorded at $\sqrt{s}=13 \mathrm{TeV}$ by the CMS detector at the LHC in 2017, corresponding to a total integrated luminosity of $41.3 \mathrm{fb}^{-1}$ [45]. Five distinct final states are considered: $Z(\nu \nu) H, W(\mu \nu) H, W(e \nu) H, Z(\mu \mu) H$, and $Z(e e) H$, corresponding to three channels with 0,1 , or 2 charged leptons from the vector boson decay. In addition, two identified jets due to hadronization of bottom quarks ( $b$ jets) from the Higgs boson decay are required. Important background processes include the production of $W$ and $Z$ bosons in association with jets ( $V+$ jets), production of top quark pairs $(t \bar{t})$ and single top quarks $(t)$, diboson $(W W$, $W Z, Z Z$ ), and multijet (QCD) events.

The analysis presented here closely follows the methods previously used to search for the $V H, H \rightarrow b \bar{b}$ process [37] and incorporates several improvements, including more 
efficient identification of $b$ jets, better dijet mass resolution, and the use of different multivariate discriminant techniques that better separate signal from background. For each channel, a signal region enriched in $\mathrm{VH}$ events is selected together with several control regions, each enriched in events from individual background processes. A simultaneous binned-likelihood fit to the shape and yield (normalization) of specific distributions for the signal and control regions for all channels combined is used to extract a possible Higgs boson signal. To validate the procedure, the same methodology is used to extract a signal for the associated production process $V Z$, with $Z \rightarrow b \bar{b}$, which has an identical final state to $V H$ with $H \rightarrow b \bar{b}$, but with a production cross section 5 to 15 times larger, depending on the kinematic regime considered. The result from this measurement is combined with those of similar measurements performed by the CMS Collaboration using 2016 data and run 1 data, in the $V H$ and other production processes. This combination leads to an observation of the Higgs boson decay to bottom quarks. An observation of this decay mode by the ATLAS Collaboration [46] was submitted for publication at the same time as this Letter.

The central feature of the CMS apparatus is a superconducting solenoid of $6 \mathrm{~m}$ internal diameter, providing a magnetic field of 3.8 T. A silicon pixel and strip tracker, a lead tungstate crystal electromagnetic calorimeter, and a brass and scintillator hadron calorimeter, each composed of a barrel and two end cap sections, reside within the solenoid. Forward calorimeters extend the pseudorapidity coverage provided by the barrel and end cap detectors. Muons are detected in gas-ionization chambers embedded in the steel flux-return yoke outside the solenoid. A more detailed description of the CMS detector, together with a definition of the coordinate system and the relevant kinematic variables, can be found in Ref. [47].

Signal and background processes are simulated with several Monte Carlo (MC) event generators, while the CMS detector response is modeled with GEANT4 [48]. The quarkinduced $\mathrm{ZH}$ and $\mathrm{WH}$ signal processes are generated at nextto-leading order (NLO) QCD accuracy using the POWHEG v2 [49-51] event generator extended with the MiNLO procedure [52,53], while the gluon-induced $Z H$ process is generated at leading order (LO) accuracy with POWHEG v2. The Higgs boson mass is set to $125 \mathrm{GeV}$ for all signal samples. Diboson background events are generated with MADGRAPH 5_AMC@NLO v2.4.2 [54] at NLO with the FxFx merging scheme [55] and up to two additional partons. The same generator is used at LO accuracy with the MLM matching scheme [56] to generate $V+$ jets events in inclusive and $b$-quark enriched configurations with up to four additional partons, and to generate a sample of QCD events. The $t \bar{t}$ [57] and single $t$ production processes in the $t W$ [58] and $t$ [59] channels are generated to NLO accuracy with POWHEG v2, while the $s$ channel [60] single $t$ process is generated with MADGRAPH 5_AMC@NLO v2.4.2.
The parton distribution functions used to produce all samples are the next-to-next-to-leading order (NNLO) NNPDF3.1 set [61]. For parton showering and hadronization, the matrix element generators are interfaced with PYTHIA v8.230 [62]. For all samples, simulated additional $p p$ interactions (pileup) are added to the hard-scattering process with the multiplicity distribution matched to the 2017 data.

The production cross sections for the signal samples are rescaled as a function of the vector boson transverse momentum, $p_{T}(V)$, to NNLO QCD + NLO electroweak accuracy combining the VHNNLO [63-66], VH@NNLO $[67,68]$, and HAWK v2.0 [69] generators as described in Ref. [32]. The production cross sections for the $t \bar{t}$ samples are rescaled to the NNLO prediction with the next-to-nextto-leading-log result obtained from ToP++ v2.0 [70], while the $V+$ jets samples are rescaled to the NNLO cross sections using FEWZ 3.1 [71]. In the $V+$ jets samples used in this analysis, the $p_{T}(V)$ spectrum in data is observed to be softer than in simulated samples, as expected from higherorder electroweak corrections to the production processes [72]. Events in each channel are reweighted to account for electroweak corrections to $p_{T}(V)$, which reach up to $10 \%$ for $p_{T}(V)$ near $400 \mathrm{GeV}$. In addition, a differential LO-toNLO correction is applied as a function of the separation in $\eta$ between the two jets from the Higgs boson decay [37]. The $t \bar{t}$ samples are reweighted as a function of top quark $p_{T}$ to account for the known difference between data and simulation [73].

The CMS particle-flow (PF) event algorithm [74] is used to reconstruct and identify individual particles (PF objects) with an optimized combination of information from the various elements of the CMS detector. This algorithm is employed at the trigger level, and in the more detailed reconstruction of data that occurs off-line. The objects identified by the algorithm comprise candidate electrons, muons, photons, and charged as well as neutral hadrons. Jets are reconstructed from PF objects using the anti- $k_{t}$ clustering algorithm [75] implemented in the FASTJET package [76,77], with a distance parameter of 0.4. The missing transverse momentum vector $\vec{p}_{T}^{\text {miss }}$ is defined as the negative vectorial $p_{T}$ sum of all the PF objects identified in the event, and its magnitude is referred to as $p_{T}^{\text {miss }}$.

The reconstructed vertex with the largest value of summed physics-object $p_{T}^{2}$ is considered to be the primary $p p$ interaction vertex. The physics objects used in this calculation are jets clustered using the jet finding algorithm with the tracks assigned to the vertex as inputs, and the associated missing transverse momentum computed as the negative vectorial $p_{T}$ sum of those jets. All charged hadrons that originate from pileup are removed from consideration in the event. In addition, the average neutral energy density from pileup is evaluated from PF objects and subtracted from the reconstructed jets as well as from the summed energy in the vicinity of leptons (isolation), as described in Ref. [74]. 
Events of interest are selected on-line using a two-tiered trigger system [78] based on custom hardware processors and a farm of commercial processors running a version of the full off-line reconstruction software optimized for speed. Events in the 0-lepton channel are selected primarily by a trigger requiring both $p_{T}{ }^{\text {miss }}$ and $H_{T}^{\text {miss }}$ to be larger than $120 \mathrm{GeV}$, where $H_{T}^{\text {miss }}$ is defined as the magnitude of the negative vectorial $p_{T}$ sum of all jets. Single-lepton triggers are used to select events in the 1-lepton channel, where the leptons are required to be isolated from other PF objects. The single-muon trigger requires $p_{T}>27 \mathrm{GeV}$, while the single-electron trigger requires $p_{T}>32 \mathrm{GeV}$. For the 2-lepton channel, dilepton triggers are employed with minimum $p_{T}$ requirements of $(17,8) \mathrm{GeV}$ for the two muons and $(23,12) \mathrm{GeV}$ for the two electrons. After offline requirements, the 0-lepton trigger efficiency reaches $100 \%$ above $p_{T}^{\text {miss }} \sim 220 \mathrm{GeV}$, while the 1-lepton triggers have efficiencies of approximately $95 \%$ for muons and $90 \%$ for electrons, and the dilepton trigger efficiency is approximately $91 \%$ for muons and $96 \%$ for electrons.

Events are selected off-line based on the presence of 0,1 , or 2 leptons (muons or electrons) and two identified $b$ jets. Muons and electrons from $W$ or $Z$ boson decays are identified using the selection criteria defined in Ref. [37], including stringent quality and isolation requirements. The lepton $p_{T}$ requirements are $p_{T}>25(30) \mathrm{GeV}$ for muons (electrons) in the 1-lepton channel, and $p_{T}>20 \mathrm{GeV}$ for each lepton in the 2-lepton channel. In the 0- and 1-lepton channels, events with additional isolated muons or electrons are rejected. Minimum $p_{T}$ requirements of $(60,35),(25,25)$, and $(20,20) \mathrm{GeV}$ are applied on the two jets forming the Higgs boson candidate in the 0-, 1-, and 2-lepton channels, respectively, which are also required to satisfy $|\eta|<2.5$.

The reconstruction of vector boson decays differs by channel. In the 0-lepton channel, $p_{T}{ }^{\text {miss }}$ is interpreted as the $p_{T}$ of the $Z$ boson, while in the 2-lepton channel the $Z$ boson is reconstructed directly from lepton pairs requiring an invariant mass in the range $75-105 \mathrm{GeV}$. In the 1-lepton channel, the $W$ boson candidate is reconstructed from the $\vec{p}_{T}$ of the single isolated lepton and $\vec{p}_{T}^{\text {miss }}$.

Higgs boson candidates are reconstructed from the pair of jets (" $j j$ ") in the event most likely to originate from $b$ quarks, as determined by a combined secondary vertex algorithm (deepCSV) based on a deep neural network discriminant (DNN) [79]. The deepCSV algorithm provides a continuous discriminator score combining information about tracks displaced from the primary vertex, identified secondary vertices, jet kinematic variables, and information related to the presence of soft leptons in the jet. Of the two jets forming the Higgs boson candidate, the one with a larger deepCSV score is required to satisfy a tight working point with misidentification rate of $0.1 \%$ for light quark and gluon jets, while the jet with a lower deepCSV score (DCSV2) must satisfy a loose working point with a $10 \%$ misidentification rate.
All backgrounds are substantially reduced by requiring large $p_{T}(V)$ [80]. The requirements are $p_{T}^{\text {miss }}>170 \mathrm{GeV}$ and $p_{T}(V)>150 \mathrm{GeV}$ in the 0 - and 1-lepton channels, respectively, while two regions are used in the 2-lepton channel: $50<p_{T}(V) \leq 150 \mathrm{GeV}$ and $p_{T}(V)>150 \mathrm{GeV}$. Residual backgrounds from $t \bar{t}$ and QCD processes are reduced in the 1-lepton channel by rejecting events with more than one extra jet satisfying $p_{T}>30 \mathrm{GeV}$ and $|\eta|<2.5$, and in the 0-lepton channel with additional requirements on the angular separation between $\vec{p}_{T}^{\text {miss }}$ and its nearest jet.

After all event selection criteria are applied, the resolution on the dijet invariant mass $m(j j)$ of reconstructed Higgs boson decays is approximately $15 \%$. The mass resolution is improved by applying a multivariate regression technique similar to that employed in Ref. [37], with a DNN trained on $b$ jets from simulated $t \bar{t}$ events with input variables that include several properties of any secondary vertices in the jet, as well as the energy and composition of the jet. In addition, recovery of final-state radiation is achieved by adding to the $m(j j)$ calculation the momenta of jets near to either of the Higgs boson candidate jets. In the 2-lepton channel, with no genuine $\vec{p}_{T}^{\text {miss }}$ from the hardscattering process, a kinematic fit of the entire event is performed requiring $p_{T}$ balance between the dilepton and dijet systems within the experimental uncertainty. All three improvements are validated in data by studying the $p_{T}(\ell \ell) / p_{T}(j j)$ distribution in samples of $Z \rightarrow \ell \ell$ events containing at least one $b$-tagged jet, and by studying the top quark mass distribution in a high-purity sample of $t \bar{t}$ events. After these improvements, the average resolution on $m(j j)$ is in the 10\%-13\% range, depending on the channel and the $p_{T}$ of the reconstructed Higgs boson.

For each channel, a signal region enriched in $\mathrm{VH}$ events is selected together with several control regions, each enriched in events from individual background processes. The signal regions are defined as $60<m(j j)<160 \mathrm{GeV}$ in the 0-lepton channel, and $90<m(j j)<150 \mathrm{GeV}$ in the 1- and 2-lepton channels. The score of a DNN for events in each of these signal regions, which further separates signal from background, is used in the signal extraction fit. The DNNs are trained separately for each channel using simulated samples for signal and all background processes. The set of input variables is chosen by an iterative optimization procedure from a large number of potentially discriminating variables. Among the most discriminating variables for all channels are $m(j j), p_{T}(V)$, DCSV2, the number of additional jets, and the angular separation between the two jets forming the Higgs boson candidate. Events in control regions are used in the fit to normalize the major background processes directly from data. These regions are selected for $t \bar{t}$ production (TT), and for the production of $W$ and $Z$ bosons in association with either predominantly heavy-flavor (HF) or light-flavor (LF) jets using the criteria described in Ref. [37].

The signal strength $\mu$, defined as the measured production cross section times branching fraction divided by the 
TABLE I. Major sources of uncertainty in the measurement of the signal strength $\mu$, and their observed impact $(\Delta \mu)$ from a fit to the 2017 data set, are listed. The total uncertainty is separated into four components: statistical (including data yields), experimental, MC sample size, and theory. Detailed decompositions of the statistical, experimental, and theory components are specified. The impact of each uncertainty is evaluated considering only that source. Because of correlations in the combined fit between nuisance parameters in different sources, the sum in quadrature for each source does not in general equal the total uncertainty of each component.

\begin{tabular}{lcc}
\hline \hline Uncertainty source & \multicolumn{2}{c}{$\Delta \mu$} \\
\hline Statistical & +0.26 & -0.26 \\
$\quad$ Normalization of backgrounds & +0.12 & -0.12 \\
Experimental & +0.16 & -0.15 \\
$\quad$-tagging efficiency and misid & +0.09 & -0.08 \\
$\quad$ + jets modeling & +0.08 & -0.07 \\
Jet energy scale and resolution & +0.05 & -0.05 \\
$\quad$ Lepton identification & +0.02 & -0.01 \\
$\quad$ Luminosity & +0.03 & -0.03 \\
$\quad$ Other experimental uncertainties & +0.06 & -0.05 \\
MC sample size & +0.12 & -0.12 \\
Theory & +0.11 & -0.09 \\
$\quad$ Background modeling & +0.08 & -0.08 \\
Signal modeling & +0.07 & -0.04 \\
Total & +0.35 & -0.33 \\
\hline \hline
\end{tabular}

expected SM value, is extracted from a simultaneous binned fit of the signal and control regions. The DNN score is used as the fitted variable in each signal region, while different strategies are used in the control regions. For the TT and LF control regions, only the yields of these processes are considered in the fit. For the HF control region, DCSV2 is used as the fitted variable in the 2-lepton channel, while the score of a dedicated DNN (DNNHF) is used in the 0- and 1-lepton channels. The DNNHF uses the same variables as the signal region DNN, but is trained to individually distinguish the $t \bar{t}$, single $t$, and $V+$ jets, background processes.

The significance of the observed excess of events in the fit is computed using the profile likelihood asymptotic approximation [81-84]. All results reported here are obtained for a Higgs boson mass of $m_{H}=125.09 \mathrm{GeV}$ [26]. For the 2017 data, the observed significance is $3.3 \sigma$ above the background-only hypothesis, while $3.1 \sigma$ is expected for the SM Higgs boson. The corresponding measured signal strength is $\mu=1.08 \pm 0.34$, where the uncertainty is a combination of statistical and systematic components. Table I lists the major sources of uncertainty and their observed impact on $\mu$ from the fit. Dominant systematic uncertainties arise from the background normalizations, simulated sample size, $b$-tagging efficiency and misidentification rates, and $V+$ jets modeling. All sources of systematic uncertainty are included in the fit as independent nuisance parameters.
The $V Z$ process with $Z \rightarrow b \bar{b}$, having an identical final state as the $V H$ process with $H \rightarrow b \bar{b}$, serves to validate the methodology used in the search for the latter process. To extract this diboson signal, the DNNs are trained using the simulated samples for this process as signal. All other processes, including $\mathrm{VH}$ production (at the predicted SM rate), are treated as background. The only modification made to the analysis is the requirement that the signal region is in the interval $[60,160] \mathrm{GeV}$ in $m(j j)$ for all channels. The excess of events for the combined $W Z$ and $\mathrm{ZZ}$ production processes has an observed significance of $5.2 \sigma$ from the background-only hypothesis, where $5.0 \sigma$ is expected. The corresponding observed signal strength is $\mu=1.05 \pm 0.22$.

Measurements of the $V H$ process with $H \rightarrow b \bar{b}$ reported above are combined with the results of a similar measurement performed by the CMS Collaboration using data collected at $13 \mathrm{TeV}$ in 2016 corresponding to $35.9 \mathrm{fb}^{-1}$ [37]. All systematic uncertainties are assumed to be uncorrelated in this fit, except for theory uncertainties and the dominant uncertainties in the measurement of the jet energy scale, which are assumed to be fully correlated. The run 2 (2016 and 2017 data sets) combination yields an observed signal significance of $4.4 \sigma$, where $4.2 \sigma$ is expected, and a signal strength of $\mu=1.06 \pm 0.26$.

The results $V H$ from run 2 are combined with the results of a similar CMS analysis of the run 1 data using $p p$ collisions at $\sqrt{s}=7$ and $8 \mathrm{TeV}$ with data samples corresponding to integrated luminosities of up to 5.1 and $18.9 \mathrm{fb}^{-1}$, respectively $[25,44]$. Systematic uncertainties in this fit are assumed to be uncorrelated for separate collision energies, except for the theory uncertainties. The combination yields an observed signal significance of $4.8 \sigma$, where $4.9 \sigma$ is expected. The measured signal strength is $\mu=1.01 \pm 0.22[0.17$ (stat) $\pm 0.09(\exp ) \pm 0.06(\mathrm{MC}) \pm$ 0.08 (theo)], where the decomposition of the total uncertainty into its components is specified in brackets following the definitions in Table I. Figure 1 (left) shows the distribution of events in all channels sorted according to the observed value of $\log _{10}(S / B)$ for the combined run 1 and run 2 data sets, where signal $S$ and background $B$ yields are determined from the corresponding discriminant score used in each analysis (DNNs for the 2017 data set, boosted decision trees for all other data sets). Figure 1 (right) summarizes the signal strengths for $V H$ production, with $H \rightarrow b \bar{b}$, separately for the different data sets and the combination, while Table II summarizes the significances, also including a breakdown of the 2017 results separated by channel.

An alternative to fitting the DNN score is to fit the $m(j j)$ distribution, which results in less sensitivity but enables a more direct visualization of the Higgs boson signal. As in the $V Z$ analysis, the signal region is defined to be in the interval $[60,160] \mathrm{GeV}$ in $m(j j)$. This study is performed only with the 2016 and 2017 data sets, in which events are 

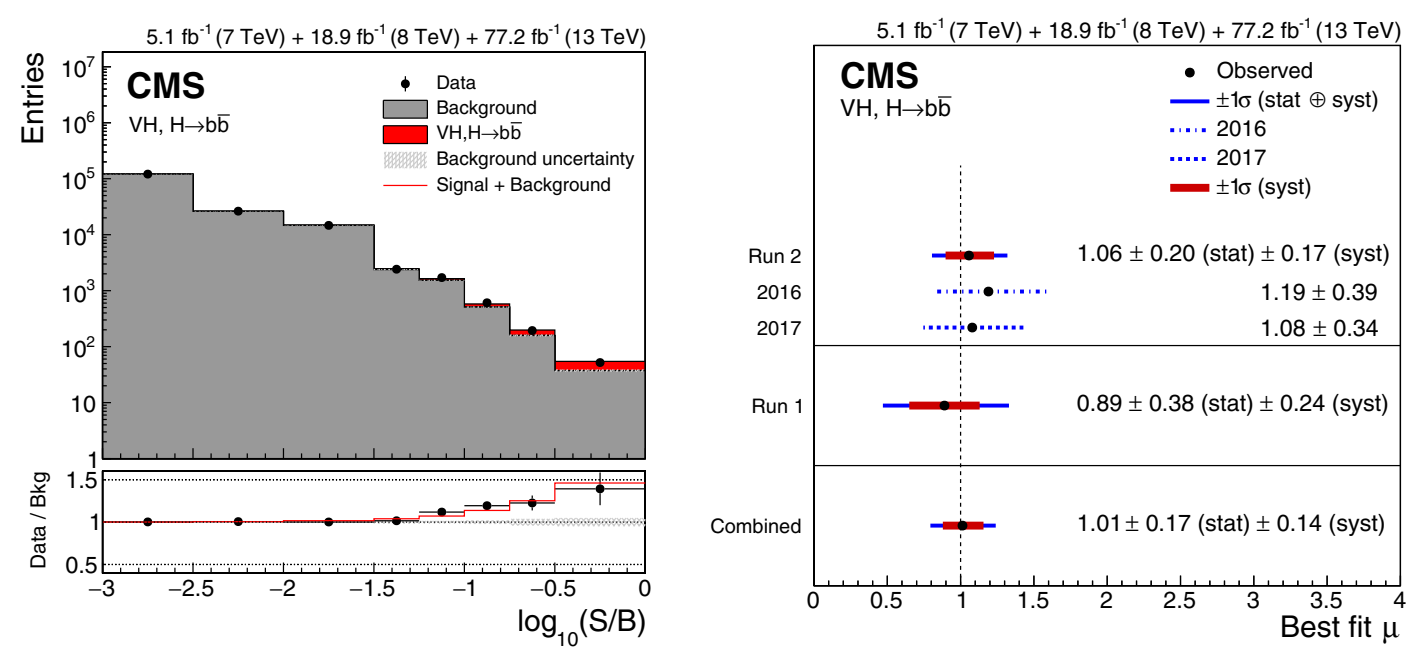

FIG. 1. Left: distributions of signal, background, and data event yields sorted into bins of similar signal-to-background ratio, as given by the result of the fit to their corresponding multivariate discriminant. All events in the $V H, H \rightarrow b \bar{b}$ signal regions of the combined run 1 and run 2 data sets are included. The red histogram indicates the Higgs boson signal contribution, while the gray histogram is the sum of all background yields. The bottom panel shows the ratio of the data to the background, with the total uncertainty in the background yield indicated by the gray hatching. The red line indicates the sum of signal plus background contribution divided by the background yield. Right: best-fit value of the signal strength $\mu$, at $m_{H}=125.09 \mathrm{GeV}$, for the fit of all $V H, H \rightarrow b \bar{b}$ channels in the run 1 and run 2 data sets. Also shown are the individual results of the 2016 and 2017 measurements, the run 2 combination, and the run 1 result. Horizontal error bars indicate the $1 \sigma$ systematic (red) and $1 \sigma$ total (blue) uncertainties, and the vertical dashed line indicates the SM expectation.

categorized into four bins of increasing signal-to-background ratio according to the score of their corresponding discriminant, obtained with those input variables correlated with $m(j j)$ fixed to their mean values. The resulting four $m(j j)$ distributions in each data set are fit together with the same distributions used in the control regions, described above, to extract signal and background yields. The fitted $m(j j)$ distributions are combined and weighted by

TABLE II. Expected and observed significances, in $\sigma$, and observed signal strengths for the $V H$ production process with $H \rightarrow b \bar{b}$. Results are shown separately for 2017 data, combined run 2 (2016 and 2017) data, and for the combination of the run 1 and run 2 data sets. For the 2017 analysis, results are shown separately for the individual signal strengths for each channel from a combined simultaneous fit to all channels. All results are obtained for $m_{H}=125.09 \mathrm{GeV}$ combining statistical and systematic uncertainties.

\begin{tabular}{lccc}
\hline \hline & \multicolumn{2}{c}{ Significance $(\sigma)$} & \\
\cline { 2 - 3 } Data set & Expected & Observed & Signal strength \\
\hline 2017 & & & \\
0-lepton & 1.9 & 1.3 & $0.73 \pm 0.65$ \\
1-lepton & 1.8 & 2.6 & $1.32 \pm 0.55$ \\
2-lepton & 1.9 & 1.9 & $1.05 \pm 0.59$ \\
Combined & 3.1 & 3.3 & $1.08 \pm 0.34$ \\
Run 2 & 4.2 & 4.4 & $1.06 \pm 0.26$ \\
Run 1 run 2 & 4.9 & 4.8 & $1.01 \pm 0.22$ \\
\hline \hline
\end{tabular}

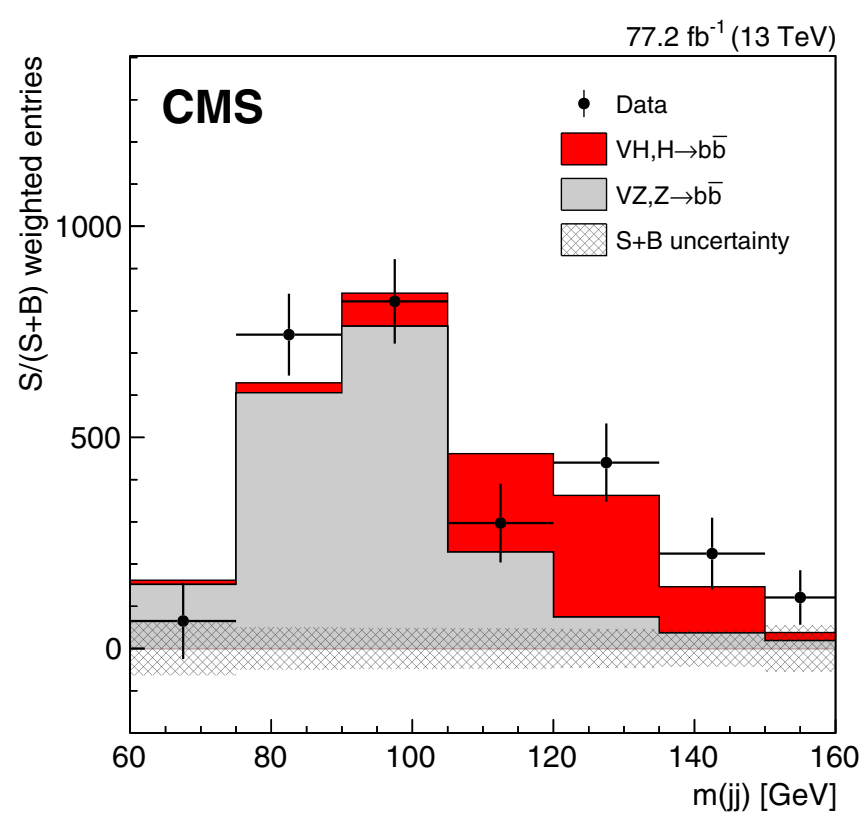

FIG. 2. Dijet invariant mass distribution for events weighted by $S /(S+B)$ in all channels combined in the 2016 and 2017 data sets. Weights are derived from a fit to the $m(j j)$ distribution, as described in the text. Shown are data (points) and the fitted $V H$ signal (red) and $V Z$ background (grey) distributions, with all other fitted background processes subtracted. The error bar for each bin represents the presubtraction $1 \sigma$ statistical uncertainty on the data, while the gray hatching indicates the $1 \sigma$ total uncertainty on the signal and all background components. 


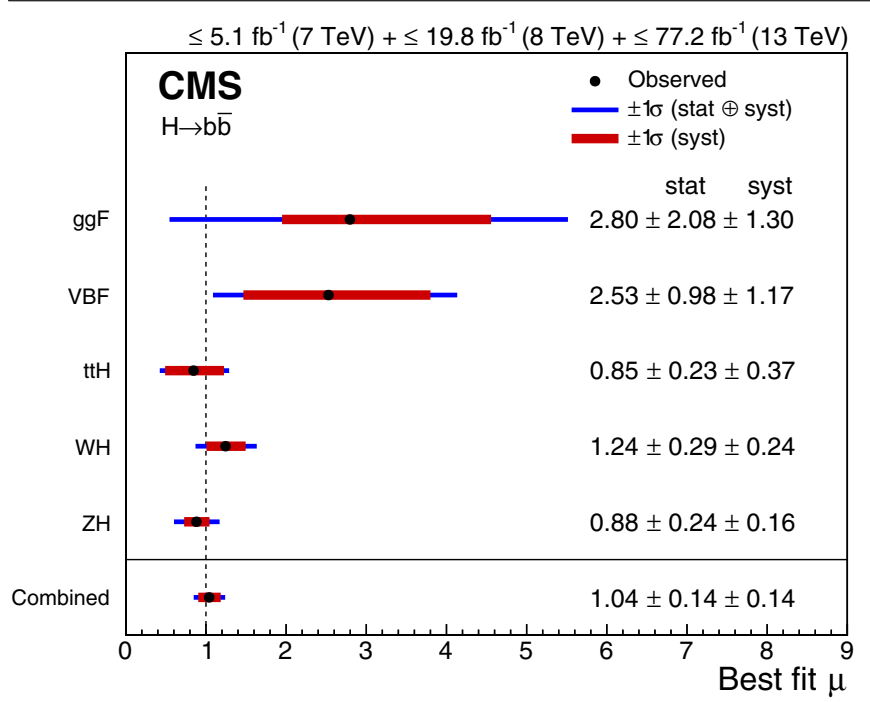

FIG. 3. Best-fit value of the $H \rightarrow b \bar{b}$ signal strength with its $1 \sigma$ systematic (red) and total (blue) uncertainties for the five individual production modes considered, as well as the overall combined result. The vertical dashed line indicates the standard model expectation. All results are extracted from a single fit combining all input analyses, with $m_{H}=125.09 \mathrm{GeV}$.

$S /(S+B)$, where $S$ and $B$ are computed from the Higgs boson signal yield and the sum of all background yields for each category considering their fitted normalizations, respectively. The resulting combined $m(j j)$ distribution, after background subtraction, is shown in Fig. 2, where the $V H$ and $V Z$ contributions are separately visible.

A combination of CMS measurements of the $H \rightarrow b \bar{b}$ decay is performed, including dedicated analyses for the following production processes: $V H$ (reported above), gluon fusion [38], vector boson fusion [44], and associated production with top quarks $[30,41,42]$. These analyses use data collected at 7, 8, and $13 \mathrm{TeV}$, depending on the process. In this fit, most sources of systematic uncertainty are treated as uncorrelated. The dominant jet energy scale uncertainties are treated as correlated between processes at the same collision energy, while the theory uncertainties are correlated between all processes and data sets. The observed (expected) signal significance is $5.6(5.5) \sigma$, and the measured signal strength is $\mu=1.04 \pm 0.20$. In addition to the overall signal strength for the $H \rightarrow b \bar{b}$ decay, the signal strengths for the individual production processes are also determined in this combination, where contributions from a single production process to multiple channels are properly accounted for in the fit. All results are summarized in Fig. 3.

In summary, measurement of the standard model Higgs boson decaying to bottom quarks has been presented. A combination of all CMS measurements of the $V H, H \rightarrow b \bar{b}$ process using proton-proton collisions recorded at center of mass energies of 7,8 , and $13 \mathrm{TeV}$, yields an observed (expected) significance of 4.8 (4.9) standard deviations at
$m_{H}=125.09 \mathrm{GeV}$, and the signal strength is $\mu=1.01 \pm$ 0.22 . Combining this result with previous measurements by the CMS Collaboration of the $H \rightarrow b \bar{b}$ decay in events where the Higgs boson is produced through gluon fusion, vector boson fusion, or in association with top quarks, the observed (expected) significance increases to 5.6 (5.5) standard deviations and the signal strength is $\mu=1.04 \pm$ 0.20 . This constitutes the observation of the $H \rightarrow b \bar{b}$ decay by the CMS Collaboration.

We congratulate our colleagues in the CERN accelerator departments for the excellent performance of the LHC and thank the technical and administrative staffs at CERN and at other CMS institutes for their contributions to the success of the CMS effort. In addition, we gratefully acknowledge the computing centers and personnel of the Worldwide LHC Computing Grid for delivering so effectively the computing infrastructure essential to our analyses. Finally, we acknowledge the enduring support for the construction and operation of the LHC and the CMS detector provided by the following funding agencies: BMBWF and FWF (Austria); FNRS and FWO (Belgium); CNPq, CAPES, FAPERJ, FAPERGS, and FAPESP (Brazil); MES (Bulgaria); CERN; CAS, MoST, and NSFC (China); COLCIENCIAS (Colombia); MSES and CSF (Croatia); RPF (Cyprus); SENESCYT (Ecuador); MoER, ERC IUT, and ERDF (Estonia); Academy of Finland, MEC, and HIP (Finland); CEA and CNRS/IN2P3 (France); BMBF, DFG, and HGF (Germany); GSRT (Greece); NKFIA (Hungary); DAE and DST (India); IPM (Iran); SFI (Ireland); INFN (Italy); MSIP and NRF (Republic of Korea); MES (Latvia); LAS (Lithuania); MOE and UM (Malaysia); BUAP, CINVESTAV, CONACYT, LNS, SEP, and UASLP-FAI (Mexico); MOS (Montenegro); MBIE (New Zealand); PAEC (Pakistan); MSHE and NSC (Poland); FCT (Portugal); JINR (Dubna); MON, RosAtom, RAS, RFBR, and NRC KI (Russia); MESTD (Serbia); SEIDI, CPAN, PCTI, and FEDER (Spain); MOSTR (Sri Lanka); Swiss Funding Agencies (Switzerland); MST (Taipei); ThEPCenter, IPST, STAR, and NSTDA (Thailand); TUBITAK and TAEK (Turkey); NASU and SFFR (Ukraine); STFC (United Kingdom); DOE and NSF (USA).

[1] ATLAS Collaboration, Observation of a new particle in the search for the standard model Higgs boson with the ATLAS detector at the LHC, Phys. Lett. B 716, 1 (2012).

[2] CMS Collaboration, Observation of a new boson at a mass of $125 \mathrm{GeV}$ with the CMS experiment at the LHC, Phys. Lett. B 716, 30 (2012).

[3] CMS Collaboration, Observation of a new boson with mass near $125 \mathrm{GeV}$ in pp collisions at $\sqrt{s}=7$ and $8 \mathrm{TeV}$, J. High Energy Phys. 06 (2013) 081.

[4] F. Englert and R. Brout, Broken symmetry and the mass of gauge vector mesons, Phys. Rev. 13, 321 (1964). 
[5] P. W. Higgs, Broken symmetries, massless particles, and gauge fields, Phys. Rev. 12, 132 (1964).

[6] P. W. Higgs, Broken symmetries and the masses of gauge bosons, Phys. Rev. 13, 508 (1964).

[7] G. S. Guralnik, C. R. Hagen, and T. W. B. Kibble, Global conservation laws and massless particles, Phys. Rev. 13, 585 (1964).

[8] P. W. Higgs, Spontaneous symmetry breakdown without massless bosons, Phys. Rev. 145, 1156 (1966).

[9] T. W. B. Kibble, Symmetry breaking in non-abelian gauge theories, Phys. Rev. 155, 1554 (1967).

[10] ATLAS Collaboration, Measurement of Higgs boson production in the diphoton decay channel in pp collisions at center-of-mass energies of 7 and $8 \mathrm{TeV}$ with the ATLAS detector, Phys. Rev. D 90, 112015 (2014).

[11] CMS Collaboration, Observation of the diphoton decay of the Higgs boson and measurement of its properties, Eur. Phys. J. C 74, 3076 (2014).

[12] ATLAS Collaboration, Measurements of Higgs boson production and couplings in the four-lepton channel in pp collisions at center-of-mass energies of 7 and $8 \mathrm{TeV}$ with the ATLAS detector, Phys. Rev. D 91, 012006 (2015).

[13] CMS Collaboration, Measurement of the properties of a Higgs boson in the four-lepton final state, Phys. Rev. D 89, 092007 (2014).

[14] ATLAS Collaboration, Observation and measurement of Higgs boson decays to $W W^{*}$ with the ATLAS detector, Phys. Rev. D 92, 012006 (2015).

[15] ATLAS Collaboration, Study of $(W / Z) H$ production and Higgs boson couplings using $H \rightarrow W W^{*}$ decays with the ATLAS detector, J. High Energy Phys. 08 (2015) 137.

[16] CMS Collaboration, Measurement of Higgs boson production and properties in the WW decay channel with leptonic final states, J. High Energy Phys. 01 (2014) 096.

[17] ATLAS Collaboration, Evidence for the Higgs-boson Yukawa coupling to tau leptons with the ATLAS detector, J. High Energy Phys. 04 (2015) 117.

[18] CMS Collaboration, Evidence for the $125 \mathrm{GeV}$ Higgs boson decaying to a pair of $\tau$ leptons, J. High Energy Phys. 05 (2014) 104.

[19] CMS Collaboration, Observation of the Higgs boson decay to a pair of $\tau$ leptons with the CMS detector, Phys. Lett. B 779, 283 (2018).

[20] CMS Collaboration, Measurements of properties of the Higgs boson decaying to a $\mathrm{W}$ boson pair in pp collisions at $\sqrt{s}=13 \mathrm{TeV}$, arXiv:1806.05246.

[21] ATLAS Collaboration, Measurements of the Higgs boson production and decay rates and coupling strengths using pp collision data at $\sqrt{s}=7$ and $8 \mathrm{TeV}$ in the ATLAS experiment, Eur. Phys. J. C 76, 6 (2016).

[22] CMS Collaboration, Precise determination of the mass of the Higgs boson and tests of compatibility of its couplings with the standard model predictions using proton collisions at 7 and 8 TeV, Eur. Phys. J. C 75, 212 (2015).

[23] CMS Collaboration, Study of the Mass and Spin-Parity of the Higgs Boson Candidate via its Decays to Z Boson Pairs, Phys. Rev. Lett. 110, 081803 (2013).

[24] ATLAS Collaboration, Evidence for the spin-0 nature of the Higgs boson using ATLAS data, Phys. Lett. B 726, 120 (2013).
[25] ATLAS and CMS Collaborations, Measurements of the Higgs boson production and decay rates and constraints on its couplings from a combined ATLAS and CMS analysis of the LHC pp collision data at $\sqrt{s}=7$ and $8 \mathrm{TeV}$, J. High Energy Phys. 08 (2016) 045.

[26] ATLAS and CMS Collaborations, Combined Measurement of the Higgs Boson Mass in pp Collisions at $\sqrt{S}=7$ and 8 Tev with the Atlas and CMS Experiments, Proceedings, Meeting of the APS Division of Particles and Fields (DPF 2015): Ann Arbor, Michigan, USA, 2015 [Phys. Rev. Lett. 114, 191803 (2015)].

[27] CMS Collaboration, Measurements of properties of the Higgs boson decaying into the four-lepton final state in pp collisions at $\sqrt{s}=13 \mathrm{TeV}$, J. High Energy Phys. 11 (2017) 047.

[28] ATLAS Collaboration, Combined measurement of differential and total cross sections in the $H \rightarrow \gamma \gamma$ and the $H \rightarrow Z Z^{*} \rightarrow 4 \ell$ decay channels at $\sqrt{s}=13 \mathrm{TeV}$ with the ATLAS detector, arXiv:1805.10197.

[29] ATLAS Collaboration, Measurement of the Higgs boson mass in the $H \rightarrow Z Z^{*} \rightarrow 4 \ell$ and $H \rightarrow \gamma \gamma$ channels with $\sqrt{s}=13 \mathrm{TeV} p p$ collisions using the ATLAS detector, Phys. Lett. B 784, 345 (2018).

[30] CMS Collaboration, Observation of $t \bar{t} H$ Production, Phys. Rev. Lett. 120, 231801 (2018).

[31] ATLAS Collaboration, Observation of Higgs boson production in association with a top quark pair at the LHC with the ATLAS detector, Phys. Lett. B 784, 173 (2018).

[32] D. de Florian et al., Handbook of LHC Higgs cross sections: 4. Deciphering the nature of the Higgs sector, CERN Report No. CERN-2017-002-M (2016).

[33] Steven Weinberg, A Model of Leptons, Phys. Rev. Lett. 19, 1264 (1967).

[34] Y. Nambu and G. Jona-Lasinio, Dynamical model of elementary particles based on an analogy with superconductivity. I, Phys. Rev. 122, 345 (1961).

[35] CDF and D0 Collaborations, Evidence for a Particle Produced in Association with Weak Bosons and Decaying to a BottomAntibottom Quark Pair in Higgs Boson Searches at the Tevatron, Phys. Rev. Lett. 109, 071804 (2012).

[36] ATLAS Collaboration, Evidence for the $H \rightarrow b \bar{b}$ decay with the ATLAS detector, J. High Energy Phys. 12 (2017) 24.

[37] CMS Collaboration, Evidence for the Higgs boson decay to a bottom quark-antiquark pair, Phys. Lett. B 780, 501 (2018).

[38] CMS Collaboration, Inclusive Search for a Highly Boosted Higgs Boson Decaying to a Bottom Quark-Antiquark Pair, Phys. Rev. Lett. 120, 071802 (2018).

[39] ATLAS Collaboration, Search for the standard model Higgs boson produced in association with top quarks and decaying into $b \bar{b}$ in pp collisions at $\sqrt{s}=8 \mathrm{TeV}$ with the ATLAS detector, Eur. Phys. J. C 75, 349 (2015).

[40] ATLAS Collaboration, Search for the standard model Higgs boson decaying into $b \bar{b}$ produced in association with top quarks decaying hadronically in pp collisions at $\sqrt{s}=$ $8 \mathrm{TeV}$ with the ATLAS detector, J. High Energy Phys. 05 (2016) 160.

[41] CMS Collaboration, Search for the standard model Higgs boson produced in association with a top-quark pair in pp collisions at the LHC, J. High Energy Phys. 05 (2013) 145. 
[42] CMS Collaboration, Search for the associated production of the higgs boson with a top-quark pair, J. High Energy Phys. 09 (2014) 087; Erratum, J. High Energy Phys. 10 (2014) 106.

[43] ATLAS Collaboration, Search for the standard model Higgs boson produced by vector-boson fusion and decaying to bottom quarks in $\sqrt{s}=8 \mathrm{TeV}$ pp collisions with the ATLAS detector, J. High Energy Phys. 11 (2016) 112.

[44] CMS Collaboration, Search for the standard model Higgs boson produced through vector boson fusion and decaying to $b \bar{b}$, Phys. Rev. D 92, 032008 (2015).

[45] CMS Collaboration (CMS), CMS luminosity measurement for the 2017 data-taking period at $\sqrt{s}=13 \mathrm{TeV}$, CMS Physics Analysis Summary Report No. CMS-PAS-LUM17-004 (2018), https://cds.cern.ch/record/2621960.

[46] ATLAS Collaboration, Observation of $H \rightarrow b \bar{b}$ decays and $V H$ production with the ATLAS detector, arXiv:1808.08238 [Phys. Lett. B (to be published)].

[47] CMS Collaboration, The CMS experiment at the CERN LHC, J. Instrum. 3, S08004 (2008).

[48] S. Agostinelli et al. (GEANT4), GEANT4-A simulation toolkit, Nucl. Instrum. Methods Phys. Res., Sect. A 506, 250 (2003).

[49] P. Nason, A new method for combining NLO QCD with shower Monte Carlo algorithms, J. High Energy Phys. 11 (2004) 040.

[50] S. Frixione, P. Nason, and C. Oleari, Matching NLO QCD computations with parton shower simulations: the POWHEG method, J. High Energy Phys. 11 (2007) 070.

[51] S. Alioli, P. Nason, C. Oleari, and E. Re, A general framework for implementing NLO calculations in shower Monte Carlo programs: the POWHEG BOX, J. High Energy Phys. 06 (2010) 043.

[52] K. Hamilton, P. Nason, and G. Zanderighi, MINLO: MultiScale Improved NLO, J. High Energy Phys. 10 (2012) 155.

[53] G. Luisoni, P. Nason, C. Oleari, and F. Tramontano, $\mathrm{HW}^{ \pm} / \mathrm{HZ}+0$ and 1 jet at NLO with the POWHEG BOX interfaced to GoSam and their merging within MiNLO, J. High Energy Phys. 10 (2013) 083.

[54] J. Alwall, R. Frederix, S. Frixione, V. Hirschi, F. Maltoni, O. Mattelaer, H. S. Shao, T. Stelzer, P. Torrielli, and M. Zaro, The automated computation of tree-level and next-to-leading order differential cross sections, and their matching to parton shower simulations, J. High Energy Phys. 07 (2014) 079.

[55] R. Frederix and S. Frixione, Merging meets matching in MC@NLO, J. High Energy Phys. 12 (2012) 061.

[56] J. Alwall, S. Höche, F. Krauss, N. Lavesson, L. Lönnblad, F. Maltoni, M. L. Mangano, M. Moretti, C. G. Papadopoulos, F. Piccinini, S. Schumann, M. Treccani, J. Winter, and M. Worek, Comparative study of various algorithms for the merging of parton showers and matrix elements in hadronic collisions, Eur. Phys. J. C 53, 473 (2008).

[57] S. Frixione, P. Nason, and G. Ridolfi, A positive-weight next-to-leading-order Monte Carlo for heavy flavour hadroproduction, J. High Energy Phys. 09 (2007) 126.

[58] E. Re, Single-top Wt-channel production matched with parton showers using the POWHEG method, Eur. Phys. J. C 71, 11547 (2011).

[59] R. Frederix, E. Re, and P. Torrielli, Single-top t-channel hadroproduction in the four-flavour scheme with POWHEG and aMC@NLO, J. High Energy Phys. 09 (2012) 130.
[60] S. Alioli, P. Nason, C. Oleari, and E. Re, NLO single-top production matched with shower in POWHEG: $s$ - and t-channel contributions, J. High Energy Phys. 09 (2009) 111; S. Alioli, P. Nason, C. Oleari, and E. Re, Erratum, J. High Energy Phys.02 (2010) 011.

[61] R. D. Ball et al. (NNPDF Collaboration), Parton distributions from high-precision collider data, Eur. Phys. J. C 77, 663 (2017).

[62] T. Sjöstrand, S. Ask, J. R. Christiansen, R. Corke, N. Desai, P. Ilten, S. Mrenna, S. Prestel, C. O. Rasmussen, and P.Z. Skands, An Introduction to PYTHIA 8.2, Comput. Phys. Commun. 191, 159 (2015).

[63] G. Ferrera, M. Grazzini, and F. Tramontano, Higher-order QCD effects for associated WH production and decay at the LHC, J. High Energy Phys. 04 (2014) 039.

[64] G. Ferrera, M. Grazzini, and F. Tramontano, Associated ZH production at hadron colliders: the fully differential NNLO QCD calculation, Phys. Lett. B 740, 51 (2015).

[65] G. Ferrera, M. Grazzini, and F. Tramontano, Associated WH Production at Hadron Colliders: A Fully Exclusive QCD Calculation at NNLO, Phys. Rev. Lett. 107, 152003 (2011).

[66] G. Ferrera, G. Somogyi, and F. Tramontano, Associated production of a Higgs boson decaying into bottom quarks at the LHC in full NNLO QCD, Phys. Lett. B 780, 346 (2018).

[67] O. Brein, R. V. Harlander, and T. J. E. Zirke, vh@nnloHiggs Strahlung at hadron colliders, Comput. Phys. Commun. 184, 998 (2013).

[68] R. V. Harlander, S. Liebler, and T. Zirke, Higgs strahlung at the large hadron collider in the 2-higgs-doublet model, J. High Energy Phys. 02 (2014) 023.

[69] A. Denner, S. Dittmaier, S. Kallweit, and A. Mück, HAWK 2.0: A Monte Carlo program for Higgs production in vectorboson fusion and Higgs strahlung at hadron colliders, Comput. Phys. Commun. 195, 161 (2015).

[70] M. Czakon and A. Mitov, Top++: A program for the calculation of the top-pair cross-section at hadron colliders, Comput. Phys. Commun. 185, 2930 (2014).

[71] Y. Li and F. Petriello, Combining QCD and electroweak corrections to dilepton production in FEWZ, Phys. Rev. D 86, 094034 (2012).

[72] S. Kallweit, J. M. Lindert, P. Maierhöfer, S. Pozzorini, and M. Schönherr, NLO QCD + EW predictions for $V+$ jets including off-shell vector-boson decays and multijet merging, J. High Energy Phys. 04 (2016) 021.

[73] CMS Collaboration, Measurement of differential cross sections for top quark pair production using the lepton + jets final state in proton-proton collisions at $13 \mathrm{TeV}$, Phys. Rev. D 95, 092001 (2017).

[74] CMS Collaboration, Particle-flow reconstruction and global event description with the CMS detector, J. Instrum. 12, P10003 (2017).

[75] M. Cacciari, G. P. Salam, and G. Soyez, The anti- $k_{T}$ jet clustering algorithm, J. High Energy Phys. 04 (2008) 063.

[76] M. Cacciari, G. P. Salam, and G. Soyez, FastJet user manual, Eur. Phys. J. C 72, 1896 (2012).

[77] M. Cacciari and G. P. Salam, Dispelling the $N^{3}$ myth for the $k_{t}$ jet-finder, Phys. Lett. B 641, 57 (2006). 
[78] CMS Collaboration, The CMS trigger system, J. Instrum. 12, P01020 (2017).

[79] CMS Collaboration, Identification of heavy-flavour jets with the CMS detector in pp collisions at $13 \mathrm{TeV}$, J. Instrum. 13, P05011 (2018).

[80] J. M. Butterworth, A. R. Davison, M. Rubin, and G. P. Salam, Jet Substructure as a New Higgs Search Channel at the LHC, Phys. Rev. Lett. 100, 242001 (2008).

[81] T. Junk, Confidence level computation for combining searches with small statistics, Nucl. Instrum. Methods Phys. Res., Sect. A 434, 435 (1999).
[82] A. L. Read, Presentation of search results: The $C L_{s}$ technique, J. Phys. G 28, 2693 (2002).

[83] ATLAS and CMS Collaborations, LHC Higgs Combination Group, Procedure for the LHC Higgs boson search combination in Summer 2011, CERN Report No. ATL-PHYS-PUB 011-11/CMS NOTE 2011-005 (2011), http://cdsweb.cern.ch/record/1379837.

[84] G. Cowan, K. Cranmer, E. Gross, and O. Vitells, Asymptotic formulae for likelihood-based tests of new physics, Eur. Phys. J. C 71, 1554 (2011); Erratum, Eur. Phys. J. C73, 2501E (2013).

A. M. Sirunyan, ${ }^{1}$ A. Tumasyan, ${ }^{1}$ W. Adam, ${ }^{2}$ F. Ambrogi, ${ }^{2}$ E. Asilar, ${ }^{2}$ T. Bergauer, ${ }^{2}$ J. Brandstetter, ${ }^{2}$ M. Dragicevic, ${ }^{2}$ J. Erö, ${ }^{2}$ A. Escalante Del Valle, ${ }^{2}$ M. Flechl, ${ }^{2}$ R. Frühwirth, ${ }^{2, b}$ V. M. Ghete, ${ }^{2}$ J. Hrubec, ${ }^{2}$ M. Jeitler, ${ }^{2, b}$ N. Krammer, ${ }^{2}$ I. Krätschmer, ${ }^{2}$ D. Liko, ${ }^{2}$ T. Madlener, ${ }^{2}$ I. Mikulec, ${ }^{2}$ N. Rad, ${ }^{2}$ H. Rohringer, ${ }^{2}$ J. Schieck ${ }^{2, b}$ R. Schöfbeck, ${ }^{2}$ M. Spanring, ${ }^{2}$ D. Spitzbart, ${ }^{2}$ W. Waltenberger, ${ }^{2}$ J. Wittmann, ${ }^{2}$ C.-E. Wulz, ${ }^{2, b}$ M. Zarucki, ${ }^{2}$ V. Chekhovsky, ${ }^{3}$ V. Mossolov, ${ }^{3}$ J. Suarez Gonzalez, ${ }^{3}$

E. A. De Wolf, ${ }^{4}$ D. Di Croce, ${ }^{4}$ X. Janssen, ${ }^{4}$ J. Lauwers, ${ }^{4}$ M. Pieters, ${ }^{4}$ H. Van Haevermaet, ${ }^{4}$ P. Van Mechelen, ${ }^{4}$

N. Van Remortel, ${ }^{4}$ S. Abu Zeid, ${ }^{5}$ F. Blekman, ${ }^{5}$ J. D'Hondt, ${ }^{5}$ J. De Clercq, ${ }^{5}$ K. Deroover, ${ }^{5}$ G. Flouris, ${ }^{5}$ D. Lontkovskyi, ${ }^{5}$ S. Lowette, ${ }^{5}$ I. Marchesini, ${ }^{5}$ S. Moortgat, ${ }^{5}$ L. Moreels, ${ }^{5}$ Q. Python, ${ }^{5}$ K. Skovpen, ${ }^{5}$ S. Tavernier, ${ }^{5}$ W. Van Doninck, ${ }^{5}$ P. Van Mulders, ${ }^{5}$ I. Van Parijs, ${ }^{5}$ D. Beghin, ${ }^{6}$ B. Bilin, ${ }^{6}$ H. Brun, ${ }^{6}$ B. Clerbaux, ${ }^{6}$ G. De Lentdecker, ${ }^{6}$ H. Delannoy, ${ }^{6}$ B. Dorney, ${ }_{6}^{6}$ G. Fasanella, ${ }^{6}$ L. Favart ${ }^{6}$ R. Goldouzian, ${ }^{6}$ A. Grebenyuk, ${ }^{6}$ A. K. Kalsi, ${ }^{6}$ T. Lenzi, ${ }^{6}$ J. Luetic, ${ }^{6}$ N. Postiau, ${ }^{6}$ E. Starling, ${ }^{6}$ L. Thomas, ${ }^{6}$ C. Vander Velde, ${ }^{6}$ P. Vanlaer, ${ }^{6}$ D. Vannerom, ${ }^{6}$ Q. Wang, ${ }^{6}$ T. Cornelis, ${ }^{7}$ D. Dobur, ${ }^{7}$ A. Fagot, ${ }^{7}$ M. Gul, ${ }^{7}$ I. Khvastunov, ${ }^{7, c}$ D. Poyraz, ${ }^{7}$ C. Roskas, ${ }^{7}$ D. Trocino, ${ }^{7}$ M. Tytgat, ${ }^{7}$ W. Verbeke, ${ }^{7}$ B. Vermassen, ${ }^{7}$ M. Vit, ${ }^{7}$ N. Zaganidis, ${ }^{7}$ H. Bakhshiansohi, ${ }^{8}$ O. Bondu, ${ }^{8}$ S. Brochet, ${ }^{8}$ G. Bruno, ${ }^{8}$ C. Caputo, ${ }^{8}$ P. David, ${ }^{8}$ C. Delaere, ${ }^{8}$ M. Delcourt, ${ }^{8}$ A. Giammanco, ${ }^{8}$ G. Krintiras, ${ }^{8}$ V. Lemaitre ${ }^{8}$ A. Magitteri, ${ }^{8}$ K. Piotrzkowski,${ }^{8}$ A. Saggio, ${ }^{8}$ M. Vidal Marono, ${ }^{8}$ P. Vischia, ${ }^{8}$ S. Wertz,${ }^{8}$ J. Zobec, ${ }^{8}$ F. L. Alves, ${ }^{9}$ G. A. Alves, ${ }^{9}$ M. Correa Martins Junior, ${ }^{9}$ G. Correia Silva, ${ }^{9}$ C. Hensel, ${ }^{9}$ A. Moraes, ${ }^{9}$ M. E. Pol,${ }^{9}$ P. Rebello Teles, ${ }^{9}$ E. Belchior Batista Das Chagas, ${ }^{10}$ W. Carvalho, ${ }^{10}$ J. Chinellato, ${ }^{10, \mathrm{~d}}$ E. Coelho, ${ }^{10}$ E. M. Da Costa, ${ }^{10}$ G. G. Da Silveira, ${ }^{10, e}$ D. De Jesus Damiao, ${ }^{10}$ C. De Oliveira Martins, ${ }^{10}$ S. Fonseca De Souza, ${ }^{10}$ H. Malbouisson, ${ }^{10}$ D. Matos Figueiredo, ${ }^{10}$ M. Melo De Almeida, ${ }^{10}$ C. Mora Herrera, ${ }^{10}$ L. Mundim, ${ }^{10}$ H. Nogima, ${ }^{10}$ W. L. Prado Da Silva, ${ }^{10}$ L. J. Sanchez Rosas, ${ }^{10}$ A. Santoro, ${ }^{10}$ A. Sznajder,${ }^{10}$ M. Thiel,${ }^{10}$ E. J. Tonelli Manganote, ${ }^{10, d}$ F. Torres Da Silva De Araujo, ${ }^{10}$ A. Vilela Pereira, ${ }^{10}$ S. Ahuja, ${ }^{11 a}$ C. A. Bernardes,${ }^{11 a}$ L. Calligaris,${ }^{11 a}$

T. R. Fernandez Perez Tomei ${ }^{11 \mathrm{a}}$ E. M. Gregores, ${ }^{11 \mathrm{a}, 11 \mathrm{~b}}$ P. G. Mercadante,${ }^{11 \mathrm{a}, 11 \mathrm{~b}}$ S. F. Novaes, ${ }^{1 \mathrm{a} a}$ Sandra S. Padula, ${ }^{11 \mathrm{a}}$ A. Aleksandrov, ${ }^{12}$ R. Hadjiiska, ${ }^{12}$ P. Iaydjiev, ${ }^{12}$ A. Marinov, ${ }^{12}$ M. Misheva, ${ }^{12}$ M. Rodozov, ${ }^{12}$ M. Shopova, ${ }^{12}$ G. Sultanov, ${ }^{12}$ A. Dimitrov, ${ }^{13}$ L. Litov, ${ }^{13}$ B. Pavlov,${ }^{13}$ P. Petkov ${ }^{13}$ W. Fang, ${ }^{14, f}$ X. Gao, ${ }^{14, f}$ L. Yuan,${ }^{14}$ M. Ahmad, ${ }^{15}$ J. G. Bian, ${ }^{15}$ G. M. Chen, ${ }^{15}$ H. S. Chen, ${ }^{15}$ M. Chen, ${ }^{15}$ Y. Chen, ${ }^{15}$ C. H. Jiang, ${ }^{15}$ D. Leggat,${ }^{15}$ H. Liao, ${ }^{15}$ Z. Liu, ${ }^{15}$ S. M. Shaheen, ${ }^{15, g}$ A. Spiezia, ${ }^{15}$ J. Tao, ${ }^{15}$ Z. Wang, ${ }^{15}$ E. Yazgan, ${ }^{15}$ H. Zhang, ${ }^{15}$ S. Zhang, ${ }^{15, g}$ J. Zhao, ${ }^{15}$ Y. Ban, ${ }^{16}$ G. Chen, ${ }^{16}$ A. Levin, ${ }^{16}$ J. Li ${ }^{16}$ L. Li, ${ }^{16}$ Q. Li ${ }^{16}$ Y. Mao, ${ }^{16}$ S. J. Qian, ${ }^{16}$ D. Wang,${ }^{16}$ Y. Wang,${ }^{17}$ C. Avila, ${ }^{18}$ A. Cabrera, ${ }^{18}$ C. A. Carrillo Montoya, ${ }^{18}$ L. F. Chaparro Sierra ${ }^{18}$ C. Florez, ${ }^{18}$ C. F. González Hernández, ${ }^{18}$ M. A. Segura Delgado, ${ }^{18}$ B. Courbon, ${ }^{19}$ N. Godinovic, ${ }^{19}$ D. Lelas, ${ }^{19}$ I. Puljak, ${ }^{19}$ T. Sculac, ${ }^{19}$ Z. Antunovic, ${ }^{20}$ M. Kovac, ${ }^{20}$ V. Brigljevic,${ }^{21}$ D. Ferencek, ${ }^{21}$ K. Kadija, ${ }^{21}$ B. Mesic, ${ }^{21}$ M. Roguljic, ${ }^{21}$ A. Starodumov, ${ }^{21, h}$ T. Susa, ${ }^{21}$ M. W. Ather, ${ }^{22}$ A. Attikis, ${ }^{22}$ M. Kolosova, ${ }^{22}$ G. Mavromanolakis,${ }^{22}$ J. Mousa, ${ }^{22}$ C. Nicolaou, ${ }^{22}$ F. Ptochos, ${ }^{22}$ P. A. Razis, ${ }^{22}$ H. Rykaczewski, ${ }^{22}$ M. Finger, ${ }^{23, i}$ M. Finger Jr., ${ }^{23, i}$ E. Ayala,,${ }^{24}$ E. Carrera Jarrin,,${ }^{25}$ H. Abdalla, ${ }^{26, j}$ A. A. Abdelalim, ${ }^{26, k, 1}$ M. A. Mahmoud ${ }^{26, m, n}$ S. Bhowmik, ${ }^{27}$ A. Carvalho Antunes De Oliveira, ${ }^{27}$

R. K. Dewanjee, ${ }^{27}$ K. Ehataht, ${ }^{27}$ M. Kadastik, ${ }^{27}$ M. Raidal, ${ }^{27}$ C. Veelken, ${ }^{27}$ P. Eerola, ${ }^{28}$ H. Kirschenmann, ${ }^{28}$ J. Pekkanen, ${ }^{28}$ M. Voutilainen, ${ }^{28}$ J. Havukainen, ${ }^{29}$ J. K. Heikkilä, ${ }^{29}$ T. Järvinen, ${ }^{29}$ V. Karimäki, ${ }^{29}$ R. Kinnunen, ${ }^{29}$ T. Lampén, ${ }^{29}$ K. Lassila-Perini, ${ }^{29}$ S. Laurila, ${ }^{29}$ S. Lehti, ${ }^{29}$ T. Lindén, ${ }^{29}$ P. Luukka, ${ }^{29}$ T. Mäenpää, ${ }^{29}$ H. Siikonen, ${ }^{29}$ E. Tuominen, ${ }^{29}$ J. Tuominiemi, ${ }^{29}$ T. Tuuva ${ }^{30}$ M. Besancon, ${ }^{31}$ F. Couderc, ${ }^{31}$ M. Dejardin, ${ }^{31}$ D. Denegri, ${ }^{31}$ J. L. Faure, ${ }^{31}$ F. Ferri, ${ }^{31}$ S. Ganjour, ${ }^{31}$ A. Givernaud, ${ }^{31}$ P. Gras, ${ }^{31}$ G. Hamel de Monchenault, ${ }^{31}$ P. Jarry, ${ }^{31}$ C. Leloup ${ }^{31}$ E. Locci, ${ }^{31}$ J. Malcles, ${ }^{31}$ G. Negro, ${ }^{31}$ J. Rander, ${ }^{31}$ A. Rosowsky, ${ }^{31}$ M. Ö. Sahin, ${ }^{31}$ M. Titov, ${ }^{31}$ A. Abdulsalam, ${ }^{32,0}$ C. Amendola, ${ }^{32}$ I. Antropov, ${ }^{32}$ F. Beaudette, ${ }^{32}$ P. Busson, ${ }^{32}$ C. Charlot,${ }^{32}$ R. Granier de Cassagnac, ${ }^{32}$ I. Kucher, ${ }^{32}$ A. Lobanov, ${ }^{32}$ J. Martin Blanco, ${ }^{32}$ 
C. Martin Perez, ${ }^{32}$ M. Nguyen, ${ }^{32}$ C. Ochando, ${ }^{32}$ G. Ortona, ${ }^{32}$ P. Paganini, ${ }^{32}$ P. Pigard, ${ }^{32}$ J. Rembser, ${ }^{32}$ R. Salerno, ${ }^{32}$ J. B. Sauvan, ${ }^{32}$ Y. Sirois, ${ }^{32}$ A. G. Stahl Leiton, ${ }^{32}$ A. Zabi, ${ }^{32}$ A. Zghiche, ${ }^{32}$ J.-L. Agram, ${ }^{33, p}$ J. Andrea, ${ }^{33}$ D. Bloch, ${ }^{33}$ J.-M. Brom, ${ }^{33}$ E. C. Chabert, ${ }^{33}$ V. Cherepanov ${ }^{33}$ C. Collard, ${ }^{33}$ E. Conte, ${ }^{33, p}$ J.-C. Fontaine, ${ }^{33, p}$ D. Gelé, ${ }^{33}$ U. Goerlach, ${ }^{33}$ M. Jansová, ${ }^{33}$ A.-C. Le Bihan, ${ }^{33}$ N. Tonon, ${ }^{33}$ P. Van Hove, ${ }^{33}$ S. Gadrat, ${ }^{34}$ S. Beauceron, ${ }^{35}$ C. Bernet,${ }^{35}$ G. Boudoul, ${ }^{35}$ N. Chanon, ${ }^{35}$ R. Chierici, ${ }^{35}$ D. Contardo, ${ }^{35}$ P. Depasse, ${ }^{35}$ H. El Mamouni, ${ }^{35}$ J. Fay,${ }^{35}$ L. Finco,${ }^{35}$ S. Gascon, ${ }^{35}$

M. Gouzevitch, ${ }^{35}$ G. Grenier, ${ }^{35}$ B. Ille, ${ }^{35}$ F. Lagarde,${ }^{35}$ I. B. Laktineh, ${ }^{35}$ H. Lattaud,${ }^{35}$ M. Lethuillier, ${ }^{35}$ L. Mirabito, ${ }^{35}$ S. Perries, ${ }^{35}$ A. Popov, ${ }^{35, q}$ V. Sordini,${ }^{35}$ G. Touquet, ${ }^{35}$ M. Vander Donckt, ${ }^{35}$ S. Viret,${ }^{35}$ A. Khvedelidze, ${ }^{36, i}$ Z. Tsamalaidze, ${ }^{37, i}$ C. Autermann, ${ }^{38}$ L. Feld,${ }^{38}$ M. K. Kiesel, ${ }^{38}$ K. Klein, ${ }^{38}$ M. Lipinski, ${ }^{38}$ M. Preuten ${ }^{38}$ M. P. Rauch, ${ }^{38}$ C. Schomakers,${ }^{38}$ J. Schulz, ${ }^{38}$ M. Teroerde, ${ }^{38}$ B. Wittmer, ${ }^{38}$ A. Albert, ${ }^{39}$ D. Duchardt, ${ }^{39}$ M. Erdmann, ${ }^{39}$ S. Erdweg, ${ }^{39}$ T. Esch, ${ }^{39}$ R. Fischer, ${ }^{39}$ S. Ghosh, ${ }^{39}$ A. Güth, ${ }^{39}$ T. Hebbeker, ${ }^{39}$ C. Heidemann, ${ }^{39}$ K. Hoepfner, ${ }^{39}$ H. Keller, ${ }^{39}$ L. Mastrolorenzo, ${ }^{39}$ M. Merschmeyer, ${ }^{39}$ A. Meyer, ${ }^{39}$ P. Millet, ${ }^{39}$ S. Mukherjee, ${ }^{39}$ T. Pook, ${ }^{39}$ M. Radziej, ${ }^{39}$ H. Reithler, ${ }^{39}$ M. Rieger, ${ }^{39}$ A. Schmidt,${ }^{39}$ D. Teyssier,${ }^{39}$ S. Thüer ${ }^{39}$ G. Flügge, ${ }^{40}$ O. Hlushchenko, ${ }^{40}$ T. Kress, ${ }^{40}$ T. Müller, ${ }^{40}$ A. Nehrkorn ${ }^{40}$ A. Nowack, ${ }^{40}$ C. Pistone,${ }^{40}$ O. Pooth,${ }^{40}$ D. Roy, ${ }^{40}$ H. Sert ${ }^{40}$ A. Stahl, ${ }^{40, r}$ M. Aldaya Martin, ${ }^{41}$ T. Arndt ${ }^{41}$ C. Asawatangtrakuldee, ${ }^{41}$ I. Babounikau ${ }^{41}$ K. Beernaert, ${ }^{41}$ O. Behnke, ${ }^{41}$ U. Behrens, ${ }^{41}$ A. Bermúdez Martínez ${ }^{41}$ D. Bertsche, ${ }^{41}$ A. A. Bin Anuar, ${ }^{41}$ K. Borras, ${ }^{41, s}$ V. Botta, ${ }^{41}$ A. Campbell, ${ }^{41}$ P. Connor, ${ }^{41}$ C. Contreras-Campana, ${ }^{41}$ V. Danilov, ${ }^{41}$ A. De Wit, ${ }^{41}$ M. M. Defranchis, ${ }^{41}$ C. Diez Pardos, ${ }^{41}$ D. Domínguez Damiani, ${ }^{41}$ G. Eckerlin, ${ }^{41}$ T. Eichhorn, ${ }^{41}$ A. Elwood, ${ }^{41}$ E. Eren, ${ }^{41}$ E. Gallo, ${ }^{41, t}$ A. Geiser, ${ }^{41}$

J. M. Grados Luyando, ${ }^{41}$ A. Grohsjean, ${ }^{41}$ M. Guthoff, ${ }^{41}$ M. Haranko, ${ }^{41}$ A. Harb,${ }^{41}$ H. Jung, ${ }^{41}$ M. Kasemann, ${ }^{41}$ J. Keaveney, ${ }^{41}$ C. Kleinwort, ${ }^{41}$ J. Knolle, ${ }^{41}$ D. Krücker, ${ }^{41}$ W. Lange, ${ }^{41}$ A. Lelek,${ }^{41}$ T. Lenz,${ }^{41}$ J. Leonard, ${ }^{41}$ K. Lipka, ${ }^{41}$ W. Lohmann,${ }^{41, u}$ R. Mankel, ${ }^{41}$ I.-A. Melzer-Pellmann, ${ }^{41}$ A. B. Meyer, ${ }^{41}$ M. Meyer, ${ }^{41}$ M. Missiroli, ${ }^{41}$ J. Mnich, ${ }^{41}$ V. Myronenko, ${ }^{41}$ S. K. Pflitsch ${ }^{41}$ D. Pitzl ${ }^{41}$ A. Raspereza, ${ }^{41}$ P. Saxena, ${ }^{41}$ P. Schütze, ${ }^{41}$ C. Schwanenberger, ${ }^{41}$ R. Shevchenko, ${ }^{41}$ A. Singh ${ }^{41}$ H. Tholen, ${ }^{41}$ O. Turkot, ${ }^{41}$ A. Vagnerini, ${ }^{41}$ G. P. Van Onsem, ${ }^{41}$ R. Walsh ${ }^{41}$ Y. Wen, ${ }^{41}$ K. Wichmann,${ }^{41}$ C. Wissing, ${ }^{41}$ O. Zenaiev, ${ }^{41}$ R. Aggleton, ${ }^{42}$ S. Bein,${ }^{42}$ L. Benato, ${ }^{42}$ A. Benecke, ${ }^{42}$ V. Blobel,,${ }^{42}$ T. Dreyer, ${ }^{42}$ A. Ebrahimi, ${ }^{42}$ E. Garutti, ${ }^{42}$ D. Gonzalez, ${ }^{42}$ P. Gunnellini, ${ }^{42}$ J. Haller, ${ }^{42}$ A. Hinzmann, ${ }^{42}$ A. Karavdina, ${ }^{42}$ G. Kasieczka, ${ }^{42}$ R. Klanner, ${ }^{42}$ R. Kogler, ${ }^{42}$ N. Kovalchuk, ${ }^{42}$ S. Kurz, ${ }^{42}$ V. Kutzner, ${ }^{42}$ J. Lange, ${ }^{42}$ D. Marconi, ${ }^{42}$ J. Multhaup, ${ }^{42}$ M. Niedziela, ${ }^{42}$ C. E. N. Niemeyer, ${ }^{42}$ D. Nowatschin, ${ }^{42}$ A. Perieanu, ${ }^{42}$ A. Reimers, ${ }^{42}$ O. Rieger, ${ }^{42}$ C. Scharf, ${ }^{42}$ P. Schleper, ${ }^{42}$ S. Schumann, ${ }^{42}$ J. Schwandt, ${ }^{42}$ J. Sonneveld, ${ }^{42}$ H. Stadie, ${ }^{42}$ G. Steinbrück, ${ }^{42}$ F. M. Stober, ${ }^{42}$ M. Stöver ${ }^{42}$ B. Vormwald, ${ }^{42}$ I. Zoi, ${ }^{42}$ M. Akbiyik, ${ }^{43}$ C. Barth, ${ }^{43}$

M. Baselga, ${ }^{43}$ S. Baur, ${ }^{43}$ E. Butz, ${ }^{43}$ R. Caspart ${ }^{43}$ T. Chwalek, ${ }^{43}$ F. Colombo, ${ }^{43}$ W. De Boer, ${ }^{43}$ A. Dierlamm, ${ }^{43}$

K. El Morabit, ${ }^{43}$ N. Faltermann, ${ }^{43}$ B. Freund, ${ }^{43}$ M. Giffels, ${ }^{43}$ M. A. Harrendorf, ${ }^{43}$ F. Hartmann, ${ }^{43, r}$ S. M. Heindl, ${ }^{43}$ U. Husemann, ${ }^{43}$ I. Katkov, ${ }^{43, q}$ S. Kudella, ${ }^{43}$ S. Mitra ${ }^{43}$ M. U. Mozer, ${ }^{43}$ Th. Müller, ${ }^{43}$ M. Musich,${ }^{43}$ M. Plagge,${ }^{43}$ G. Quast, ${ }^{43}$ K. Rabbertz, ${ }^{43}$ M. Schröder, ${ }^{43}$ I. Shvetsov, ${ }^{43}$ H. J. Simonis, ${ }^{43}$ R. Ulrich, ${ }^{43}$ S. Wayand, ${ }^{43}$ M. Weber, ${ }^{43}$ T. Weiler, ${ }^{43}$

C. Wöhrmann, ${ }^{43}$ R. Wolf, ${ }^{43}$ G. Anagnostou, ${ }^{44}$ G. Daskalakis, ${ }^{44}$ T. Geralis, ${ }^{44}$ A. Kyriakis, ${ }^{44}$ D. Loukas, ${ }^{44}$ G. Paspalaki, ${ }^{44}$ A. Agapitos, ${ }^{45}$ G. Karathanasis, ${ }^{45}$ P. Kontaxakis, ${ }^{45}$ A. Panagiotou, ${ }^{45}$ I. Papavergou,${ }^{45}$ N. Saoulidou, ${ }^{45}$ E. Tziaferi ${ }^{45}$

K. Vellidis ${ }^{45}$ K. Kousouris, ${ }^{46}$ I. Papakrivopoulos,${ }^{46}$ G. Tsipolitis, ${ }^{46}$ I. Evangelou, ${ }^{47}$ C. Foudas, ${ }^{47}$ P. Gianneios,${ }^{47}$ P. Katsoulis, ${ }^{47}$ P. Kokkas, ${ }^{47}$ S. Mallios,${ }^{47}$ N. Manthos, ${ }^{47}$ I. Papadopoulos, ${ }^{47}$ E. Paradas, ${ }^{47}$ J. Strologas, ${ }^{47}$ F. A. Triantis, ${ }^{47}$ D. Tsitsonis, ${ }^{47}$ M. Bartók, ${ }^{48, v}$ M. Csanad,${ }^{48}$ N. Filipovic, ${ }^{48}$ P. Major,${ }^{48}$ M. I. Nagy, ${ }^{48}$ G. Pasztor, ${ }^{48}$ O. Surányi, ${ }^{48}$ G. I. Veres ${ }^{48}$ G. Bencze, ${ }^{49}$ C. Hajdu ${ }^{49}$ D. Horvath ${ }^{49, w}$ Á. Hunyadi ${ }^{49}$ F. Sikler, ${ }^{49}$ T. Á. Vámi, ${ }^{49}$ V. Veszpremi, ${ }^{49}$ G. Vesztergombi, ${ }^{49, a, x}$ N. Beni, ${ }^{50}$ S. Czellar, ${ }^{50}$ J. Karancsi, ${ }^{50, v}$ A. Makovec,${ }^{50}$ J. Molnar, ${ }^{50}$ Z. Szillasi, ${ }^{50}$ P. Raics,${ }^{51}$ Z. L. Trocsanyi, ${ }^{51}$ B. Ujvari, ${ }^{51}$ S. Choudhury, ${ }^{52}$ J. R. Komaragiri, ${ }^{52}$ P. C. Tiwari, ${ }^{52}$ S. Bahinipati, ${ }^{53, y}$ C. Kar, ${ }^{53}$ P. Mal, ${ }^{53}$ K. Mandal, ${ }^{53}$ A. Nayak, ${ }^{53, z}$ S. Roy Chowdhury, ${ }^{53}$ D. K. Sahoo, ${ }^{53, y}$ S. K. Swain,${ }^{53}$ S. Bansal,${ }^{54}$ S. B. Beri,${ }^{54}$ V. Bhatnagar, ${ }^{54}$ S. Chauhan, ${ }^{54}$ R. Chawla, ${ }^{54}$ N. Dhingra ${ }^{54}$ R. Gupta ${ }^{54}$ A. Kaur, ${ }^{54}$ M. Kaur, ${ }^{54}$ S. Kaur, ${ }^{54}$ P. Kumari, ${ }^{54}$ M. Lohan,${ }^{54}$ M. Meena, ${ }^{54}$ A. Mehta,${ }^{54}$ K. Sandeep, ${ }^{54}$ S. Sharma, ${ }^{54}$ J. B. Singh, ${ }^{54}$ A. K. Virdi,${ }^{54}$ G. Walia, ${ }^{54}$ A. Bhardwaj, ${ }^{55}$ B. C. Choudhary, ${ }^{55}$ R. B. Garg, ${ }^{55}$ M. Gola, ${ }^{55}$ S. Keshri, ${ }^{55}$ Ashok Kumar, ${ }^{55}$ S. Malhotra, ${ }^{55}$ M. Naimuddin, ${ }^{55}$ P. Priyanka, ${ }^{55}$ K. Ranjan, ${ }^{55}$ Aashaq Shah,${ }^{55}$ R. Sharma ${ }^{55}$ R. Bhardwaj, ${ }^{56, a a}$ M. Bharti, ${ }^{56, a a}$ R. Bhattacharya, ${ }^{56}$ S. Bhattacharya, ${ }^{56}$ U. Bhawandeep, ${ }^{56, a a}$ D. Bhowmik, ${ }^{56}$ S. Dey ${ }^{56}$ S. Dutt, ${ }^{56, \text { aa }}$ S. Dutta ${ }^{56}$ S. Ghosh,${ }^{56}$ K. Mondal, ${ }^{56}$ S. Nandan, ${ }^{56}$ A. Purohit, ${ }^{56}$ P. K. Rout, ${ }^{56}$ A. Roy,${ }^{56}$ G. Saha,${ }^{56}$ S. Sarkar, ${ }^{56}$ M. Sharan, ${ }^{56}$ B. Singh,${ }^{56, a a}$ S. Thakur, ${ }^{56, a a}$ P. K. Behera, ${ }^{57}$ A. Muhammad,${ }^{57}$ R. Chudasama, ${ }^{58}$ D. Dutta, ${ }^{58}$ V. Jha, ${ }^{58}$ V. Kumar, ${ }^{58}$ D. K. Mishra, ${ }^{58}$ P. K. Netrakanti, ${ }^{58}$ L. M. Pant, ${ }^{58}$ P. Shukla, ${ }^{58}$ P. Suggisetti, ${ }^{58}$ T. Aziz, ${ }^{59}$ M. A. Bhat, ${ }^{59}$ S. Dugad,${ }^{59}$ G. B. Mohanty,${ }^{59}$ N. Sur ${ }^{59}$ Ravindra Kumar Verma,${ }^{59}$ S. Banerjee, ${ }^{60}$ S. Bhattacharya,${ }^{60}$ S. Chatterjee,${ }^{60}$ P. Das,${ }^{60}$ M. Guchait, ${ }^{60}$ Sa. Jain, ${ }^{60}$ S. Karmakar, ${ }^{60}$ S. Kumar, ${ }^{60}$ M. Maity, ${ }^{60, b b}$ G. Majumder, ${ }^{60}$ K. Mazumdar,${ }^{60}$ N. Sahoo, ${ }^{60}$ T. Sarkar, ${ }^{60, b b}$ S. Chauhan, ${ }^{61}$ S. Dube, ${ }^{61}$ V. Hegde, ${ }^{61}$ A. Kapoor,${ }^{61}$ K. Kothekar,${ }^{61}$ S. Pandey, ${ }^{61}$ A. Rane, ${ }^{61}$ A. Rastogi, ${ }^{61}$ 
S. Sharma, ${ }^{61}$ S. Chenarani, ${ }^{62, c c}$ E. Eskandari Tadavani, ${ }^{62}$ S. M. Etesami, ${ }^{62, c c}$ M. Khakzad ${ }^{62}$ M. Mohammadi Najafabadi, ${ }^{62}$ M. Naseri, ${ }^{62}$ F. Rezaei Hosseinabadi, ${ }^{62}$ B. Safarzadeh,${ }^{62, d d}$ M. Zeinali, ${ }^{62}$ M. Felcini, ${ }^{63}$ M. Grunewald,${ }^{63}$ M. Abbrescia,${ }^{64 a, 64 b}$ C. Calabria, ${ }^{64 a, 64 b}$ A. Colaleo, ${ }^{64 a}$ D. Creanza, ${ }^{64 a, 64 c}$ L. Cristella,,${ }^{64 a, 64 b}$ N. De Filippis, ${ }^{64 a, 64 c}$ M. De Palma, ${ }^{64 a, 64 b}$ A. Di Florio, ${ }^{64 a, 64 b}$ F. Errico, ${ }^{64 a, 64 b}$ L. Fiore,${ }^{64 a}$ A. Gelmi,${ }^{64 a, 64 b}$ G. Iaselli, ${ }^{64 a, 64 c}$ M. Ince, ${ }^{64 a, 64 b}$ S. Lezki, ${ }^{64 a, 64 b}$ G. Maggi, ${ }^{64 a, 64 c}$ M. Maggi, ${ }^{64 a}$ G. Miniello, ${ }^{64 a, 64 b}$ S. My ${ }^{64 a, 64 b}$ S. Nuzzo, ${ }^{64 a, 64 b}$ A. Pompili, ${ }^{64 a, 64 b}$ G. Pugliese,${ }^{64 a, 64 c}$ R. Radogna, ${ }^{64 a}$ A. Ranieri, ${ }^{64 a}$ G. Selvaggi, ${ }^{64 a, 64 b}$ A. Sharma, ${ }^{64 a}$ L. Silvestris, ${ }^{64 a}$ R. Venditti, ${ }^{64 a}$ P. Verwilligen, ${ }^{64 a}$ G. Abbiendi, ${ }^{65 a}$ C. Battilana, ${ }^{65 a, 65 b}$ D. Bonacorsi, ${ }^{65 a, 65 b}$ L. Borgonovi, ${ }^{65 a, 65 b}$ S. Braibant-Giacomelli, ${ }^{65 a, 65 b}$ R. Campanini, ${ }^{65 a, 65 b}$ P. Capiluppi ${ }^{65 a, 65 b}$ A. Castro, ${ }^{65 a, 65 b}$ F. R. Cavallo, ${ }^{65 a}$ S. S. Chhibra ${ }^{65 a, 65 b}$ G. Codispoti, ${ }^{65 a, 65 b}$ M. Cuffiani, ${ }^{65 a, 65 b}$ G. M. Dallavalle, ${ }^{65 a}$ F. Fabbri, ${ }^{65 a}$ A. Fanfani, ${ }^{65 a, 65 b}$ E. Fontanesi, ${ }^{65 a}$ P. Giacomelli, ${ }^{65 a}$ C. Grandi, ${ }^{65 a}$ L. Guiducci, ${ }^{65 a, 65 b}$ F. Iemmi, ${ }^{65 a, 65 b}$ S. Lo Meo, ${ }^{65 a, e e}$ S. Marcellini, ${ }^{65 a}$ G. Masetti, ${ }^{65 a}$ A. Montanari, ${ }^{65 a}$ F. L. Navarria, ${ }^{65 a, 65 b}$ A. Perrotta, ${ }^{65 a}$ F. Primavera, ${ }^{65 a, 65 b}$ A. M. Rossi ${ }^{65 a, 65 b}$ T. Rovelli, ${ }^{65 a, 65 b}$ G. P. Siroli ${ }^{65 a, 65 b}$ N. Tosi ${ }^{65 a}$ S. Albergo, ${ }^{66 a, 66 b}$ A. Di Mattia, ${ }^{66 a}$ R. Potenza, ${ }^{66,66 b}$ A. Tricomi, ${ }^{66 a, 66 b}$ C. Tuve ${ }^{66 a, 66 b}$ G. Barbagli, ${ }^{67 a}$ K. Chatterjee,${ }^{67 a 67 b}$ V. Ciulli, ${ }^{67 a, 67 b}$ C. Civinini ${ }^{67 a}$ R. D’Alessandro, ${ }^{67 a, 67 b}$ E. Focardi, ${ }^{67 a, 67 b}$ G. Latino, ${ }^{67 a}$ P. Lenzi, ${ }^{67 a, 67 b}$ M. Meschini, ${ }^{67 a}$ S. Paoletti, ${ }^{67 a}$ L. Russo, ${ }^{67 a, f f}$

G. Sguazzoni, ${ }^{67 a}$ D. Strom, ${ }^{67 a}$ L. Viliani, ${ }^{67 a}$ L. Benussi, ${ }^{68}$ S. Bianco, ${ }^{68}$ F. Fabbri, ${ }^{68}$ D. Piccolo, ${ }^{68}$ F. Ferro, ${ }^{69 a}$

R. Mulargia ${ }^{69 \mathrm{a}, 69 \mathrm{~b}}$ F. Ravera, ${ }^{69 \mathrm{a}, 69 \mathrm{~b}}$ E. Robutti, ${ }^{69 \mathrm{a}}$ S. Tosi, ${ }^{69 \mathrm{a}, 69 \mathrm{~b}}$ A. Benaglia, ${ }^{70 \mathrm{a}}$ A. Beschi, ${ }^{70 a, 70 \mathrm{~b}}$ F. Brivio, ${ }^{70 \mathrm{a}, 70 \mathrm{~b}}$ V. Ciriolo, ${ }^{70 a, 70 b, r}$ S. Di Guida, ${ }^{70 a, 70 b, r}$ M. E. Dinardo, ${ }^{70 a, 70 b}$ S. Fiorendi, ${ }^{70 a, 70 b}$ S. Gennai, ${ }^{70 a}$ A. Ghezzi,${ }^{70 a, 70 b}$ P. Govoni, ${ }^{70 a, 70 b}$ M. Malberti ${ }^{70 a, 70 b}$ S. Malvezzi, ${ }^{70 a}$ D. Menasce ${ }^{70 a}$ F. Monti, ${ }^{70 a}$ L. Moroni,${ }^{70 a}$ M. Paganoni, ${ }^{70 a, 70 b}$ D. Pedrini, ${ }^{70 a}$ S. Ragazzi, ${ }^{70 a, 70 b}$ T. Tabarelli de Fatis, ${ }^{70 a, 70 b}$ D. Zuolo, ${ }^{70 a, 70 b}$ S. Buontempo, ${ }^{71 \mathrm{a}}$ N. Cavallo, ${ }^{71 \mathrm{a}, 71 \mathrm{c}}$ A. De Iorio, ${ }^{71 \mathrm{a}, 71 \mathrm{~b}}$ A. Di Crescenzo, ${ }^{71 a, 71 b}$ F. Fabozzi, ${ }^{71 a, 71 c}$ F. Fienga, ${ }^{71 a}$ G. Galati, ${ }^{71 a}$ A. O. M. Iorio, ${ }^{71 a, 71 b}$ W. A. Khan, ${ }^{71 a}$ L. Lista, ${ }^{71 a}$ S. Meola, ${ }^{71 a, 71 d, r}$ P. Paolucci, ${ }^{71 a, r}$ C. Sciacca,${ }^{71 a, 71 b}$ E. Voevodina,${ }^{71 a, 71 b}$ P. Azzi, ${ }^{72 a}$ N. Bacchetta, ${ }^{72 a}$ D. Bisello, ${ }^{72 a, 72 b}$ A. Boletti, ${ }^{72 a, 72 b}$ A. Bragagnolo, ${ }^{72 a}$ R. Carlin, ${ }^{72 a, 72 b}$ P. Checchia, ${ }^{72 a}$ M. Dall'Osso, ${ }^{72 a, 72 b}$ P. De Castro Manzano, ${ }^{72 a}$ T. Dorigo, ${ }^{72 a}$ U. Dosselli, ${ }^{72 a}$ F. Gasparini, ${ }^{72 a, 72 b}$ U. Gasparini, ${ }^{72 a, 72 b}$ A. Gozzelino, ${ }^{72 a}$ S. Y. Hoh, ${ }^{72 a}$ S. Lacaprara, ${ }^{72 a}$ P. Lujan, ${ }^{72 a}$ M. Margoni, ${ }^{72 a, 72 b}$ A. T. Meneguzzo, ${ }^{72 a, 72 b}$ J. Pazzini,${ }^{72 a, 72 b}$ M. Presilla,${ }^{72 a, 72 b}$ P. Ronchese, ${ }^{72 a, 72 b}$ R. Rossin, ${ }^{72 a, 72 b}$ F. Simonetto, ${ }^{72 a, 72 b}$ A. Tiko, ${ }^{72 a}$ E. Torassa, ${ }^{72 a}$ M. Tosi, ${ }^{72 a, 72 b}$ M. Zanetti, ${ }^{72 a, 72 b}$ P. Zotto, ${ }^{72 a, 72 b}$ G. Zumerle, ${ }^{72 a, 72 b}$ A. Braghieri, ${ }^{73 a}$ A. Magnani, ${ }^{73 a}$ P. Montagna, ${ }^{73 a, 73 b}$ S. P. Ratti, ${ }^{73 a, 73 b}$ V. Re ${ }^{73 a}$ M. Ressegotti, ${ }^{73 a, 73 b}$ C. Riccardi, ${ }^{73 a, 73 b}$ P. Salvini, ${ }^{73 a}$ I. Vai, ${ }^{73 a, 73 b}$ P. Vitulo, ${ }^{73 a, 73 b}$ M. Biasini, ${ }^{74 a, 74 b}$ G. M. Bilei ${ }^{74 a}$ C. Cecchi, ${ }^{74 a, 74 b}$ D. Ciangottini, ${ }^{74 a, 74 b}$ L. Fanò ${ }^{74 a, 74 b}$ P. Lariccia,${ }^{74 a, 74 b}$ R. Leonardi,${ }^{74 a, 74 b}$ E. Manoni,${ }^{74 a}$ G. Mantovani,${ }^{74 a, 74 b}$ V. Mariani, ${ }^{74 a, 74 b}$ M. Menichelli, ${ }^{74 a}$ A. Rossi ${ }^{74 a, 74 b}$ A. Santocchia, ${ }^{74 a}{ }^{74 b}$ D. Spiga ${ }^{74 a}$ K. Androsov, ${ }^{75 a}$ P. Azzurri ${ }^{75 a}$ G. Bagliesi, ${ }^{75 a}$ L. Bianchini ${ }^{75 a}$ T. Boccali, ${ }^{75 a}$ L. Borrello, ${ }^{75 a}$ R. Castaldi, ${ }^{75 a}$ M. A. Ciocci, ${ }^{75 a, 75 b}$ R. Dell'Orso, ${ }^{75 a}$ G. Fedi,${ }^{75 a}$ F. Fiori, ${ }^{75 a, 75 c}$ L. Giannini,${ }^{75 a, 75 c}$ A. Giassi, ${ }^{75 a}$ M. T. Grippo, ${ }^{75 a}$ F. Ligabue,${ }^{75 a, 75 \mathrm{c}}$ E. Manca,${ }^{75 a, 75 \mathrm{c}}$ G. Mandorli, ${ }^{75 a, 75 \mathrm{c}}$ A. Messineo, ${ }^{75 a, 75 \mathrm{~b}}$ F. Palla, ${ }^{75 \mathrm{a}}$ A. Rizzi ${ }^{75 a, 75 \mathrm{~b}}$ G. Rolandi, ${ }^{75 a, g g}$ P. Spagnolo, ${ }^{75 a}$ R. Tenchini ${ }^{75 a}$ G. Tonelli, ${ }^{75 a, 75 b}$ A. Venturi, ${ }^{75 a}$ P. G. Verdini, ${ }^{75 a}$ L. Barone,${ }^{76 a, 76 b}$ F. Cavallari, ${ }^{76 a}$ M. Cipriani, ${ }^{76 a, 76 b}$ D. Del Re ${ }^{76 a, 76 b}$ E. Di Marco, ${ }^{76 a, 76 b}$ M. Diemoz, ${ }^{76 a}$ S. Gelli, ${ }^{76 a, 76 b}$ E. Longo, ${ }^{76 a, 76 b}$ B. Marzocchi, ${ }^{76 a, 76 b}$ P. Meridiani, ${ }^{76 a}$ G. Organtini, ${ }^{76 a, 76 b}$ F. Pandolfi, ${ }^{76 a}$ R. Paramatti,${ }^{76 a, 76 b}$ F. Preiato, ${ }^{76 a, 76 b}$ S. Rahatlou, ${ }^{76 a, 76 b}$ C. Rovelli, ${ }^{76 a}$ F. Santanastasio, ${ }^{76 a, 76 b}$ N. Amapane,${ }^{77 a, 77 b}$ R. Arcidiacono, ${ }^{77 a, 77 c}$ S. Argiro, ${ }^{77 a, 77 b}$ M. Arneodo, ${ }^{77 a, 77 c}$ N. Bartosik, ${ }^{77 a}$ R. Bellan, ${ }^{77,77 b}$ C. Biino, ${ }^{77 a}$ A. Cappati, ${ }^{77,77 b}$ N. Cartiglia, ${ }^{77 a}$ F. Cenna, ${ }^{77,77 b}$ S. Cometti, ${ }^{77 a}$ M. Costa, ${ }^{77 a, 77 b}$ R. Covarelli, ${ }^{77,77 b}$ N. Demaria, ${ }^{77 a}$ B. Kiani, ${ }^{77 a, 77 b}$ C. Mariotti, ${ }^{77 a}$ S. Maselli, ${ }^{77 a}$ E. Migliore, ${ }^{77 a, 77 b}$ V. Monaco, ${ }^{77 a, 77 b}$ E. Monteil, ${ }^{77 a, 77 b}$ M. Monteno, ${ }^{77 a}$ M. M. Obertino, ${ }^{77,77 b}$ L. Pacher, ${ }^{77,77 b}$ N. Pastrone, ${ }^{77 a}$ M. Pelliccioni, ${ }^{77 a}$

G. L. Pinna Angioni, ${ }^{77,77 b}$ A. Romero, ${ }^{77,77 b}$ M. Ruspa, ${ }^{77,77 \mathrm{c}}$ R. Sacchi, ${ }^{77,77 b}$ R. Salvatico, ${ }^{77 a, 77 b}$ K. Shchelina, ${ }^{77 a, 77 b}$ V. Sola ${ }^{77 a}$ A. Solano, ${ }^{77 a, 77 b}$ D. Soldi,${ }^{77 a, 77 b}$ A. Staiano, ${ }^{77 a}$ S. Belforte, ${ }^{78 a}$ V. Candelise, ${ }^{78 a, 78 b}$ M. Casarsa ${ }^{78 a}$ F. Cossutti, ${ }^{78 a}$ A. Da Rold, ${ }^{78 a, 78 b}$ G. Della Ricca, ${ }^{78 a, 78 b}$ F. Vazzoler, ${ }^{78 a}{ }^{78 b}$ A. Zanetti, ${ }^{78 a}$ D. H. Kim, ${ }^{79}$ G. N. Kim ${ }^{79}$ M. S. Kim, ${ }^{79}$ J. Lee, ${ }^{79}$ S. Lee, ${ }^{79}$ S. W. Lee, ${ }^{79}$ C. S. Moon,${ }^{79}$ Y. D. Oh, ${ }^{79}$ S. I. Pak, ${ }^{79}$ S. Sekmen, ${ }^{79}$ D. C. Son, ${ }^{79}$ Y. C. Yang, ${ }^{79}$ H. Kim, ${ }^{80}$ D. H. Moon, ${ }^{80}$ G. Oh, ${ }^{80}$ B. Francois, ${ }^{81}$ J. Goh, ${ }^{81, \text { hh }}$ T. J. Kim, ${ }^{81}$ S. Cho, ${ }^{82}$ S. Choi, ${ }^{82}$ Y. Go, ${ }^{82}$ D. Gyun, ${ }^{82}$ S. Ha, ${ }^{82}$ B. Hong, ${ }^{82}$ Y. Jo, ${ }^{82}$ K. Lee, ${ }^{82}$ K. S. Lee, ${ }^{82}$ S. Lee, ${ }^{82}$ J. Lim, ${ }^{82}$ S. K. Park, ${ }^{82}$ Y. Roh ${ }^{82}$ H. S. Kim, ${ }^{83}$ J. Almond, ${ }^{84}$ J. Kim, ${ }^{84}$ J. S. Kim, ${ }^{84}$ H. Lee, ${ }^{84}$ K. Lee ${ }^{84}$ K. Nam, ${ }^{84}$ S. B. Oh, ${ }^{84}$ B. C. Radburn-Smith ${ }^{84}$ S. h. Seo, ${ }^{84}$ U. K. Yang, ${ }^{84}$ H. D. Yoo, ${ }^{84}$ G. B. Yu, ${ }^{84}$ D. Jeon, ${ }^{85}$ H. Kim, ${ }^{85}$ J. H. Kim, ${ }^{85}$ J. S. H. Lee, ${ }^{85}$ I. C. Park ${ }^{85}$ Y. Choi, ${ }^{86}$ C. Hwang, ${ }^{86}$ J. Lee,${ }^{86}$ I. Yu, ${ }^{86}$ V. Dudenas, ${ }^{87}$ A. Juodagalvis,${ }^{87}$ J. Vaitkus, ${ }^{87}$ Z. A. Ibrahim, ${ }^{88}$ M. A. B. Md Ali, ${ }^{88, i i}$ F. Mohamad Idris, ${ }^{88, j j}$ W. A. T. Wan Abdullah, ${ }^{88}$ M. N. Yusli, ${ }^{88}$ Z. Zolkapli, ${ }^{88}$ J. F. Benitez, ${ }^{89}$ A. Castaneda Hernandez, ${ }^{89}$ J. A. Murillo Quijada, ${ }^{89}$ H. Castilla-Valdez, ${ }^{90}$

E. De La Cruz-Burelo, ${ }^{90}$ M. C. Duran-Osuna ${ }^{90}$ I. Heredia-De La Cruz,${ }^{90, k k}$ R. Lopez-Fernandez, ${ }^{90}$ J. Mejia Guisao, ${ }^{90}$ R. I. Rabadan-Trejo, ${ }^{90}$ M. Ramirez-Garcia, ${ }^{90}$ G. Ramirez-Sanchez, ${ }^{90}$ R. Reyes-Almanza, ${ }^{90}$ A. Sanchez-Hernandez, ${ }^{90}$ 
S. Carrillo Moreno, ${ }^{91}$ C. Oropeza Barrera, ${ }^{91}$ F. Vazquez Valencia, ${ }^{91}$ J. Eysermans, ${ }^{92}$ I. Pedraza, ${ }^{92}$ H. A. Salazar Ibarguen, ${ }^{92}$ C. Uribe Estrada, ${ }^{92}$ A. Morelos Pineda, ${ }^{93}$ D. Krofcheck, ${ }^{94}$ S. Bheesette, ${ }^{95}$ P. H. Butler,${ }^{95}$ A. Ahmad, ${ }^{96}$ M. Ahmad, ${ }^{96}$ M. I. Asghar, ${ }^{96}$ Q. Hassan, ${ }^{96}$ H. R. Hoorani, ${ }^{96}$ A. Saddique, ${ }^{96}$ M. A. Shah,${ }^{96}$ M. Shoaib,${ }^{96}$ M. Waqas, ${ }^{96}$ H. Bialkowska,${ }^{97}$ M. Bluj, ${ }^{97}$ B. Boimska, ${ }^{97}$ T. Frueboes,${ }^{97}$ M. Górski, ${ }^{97}$ M. Kazana, ${ }^{97}$ M. Szleper, ${ }^{97}$ P. Traczyk,${ }^{97}$ P. Zalewski, ${ }^{97}$ K. Bunkowski, ${ }^{98}$ A. Byszuk, ${ }^{98,11}$ K. Doroba, ${ }^{98}$ A. Kalinowski, ${ }^{98}$ M. Konecki, ${ }^{98}$ J. Krolikowski, ${ }^{98}$ M. Misiura, ${ }^{98}$ M. Olszewski, ${ }^{98}$ A. Pyskir, ${ }^{98}$ M. Walczak,${ }^{98}$ M. Araujo, ${ }^{99}$ P. Bargassa, ${ }^{99}$ C. Beirão Da Cruz E Silva, ${ }^{99}$ A. Di Francesco, ${ }^{99}$ P. Faccioli, ${ }^{99}$ B. Galinhas, ${ }^{99}$ M. Gallinaro, ${ }^{99}$ J. Hollar,${ }^{99}$ N. Leonardo,${ }^{99}$ J. Seixas,${ }^{99}$ G. Strong, ${ }^{99}$ O. Toldaiev,${ }^{99}$ J. Varela,${ }^{99}$ S. Afanasiev, ${ }^{100}$ P. Bunin, ${ }^{100}$ M. Gavrilenko, ${ }^{100}$ I. Golutvin, ${ }^{100}$ I. Gorbunov, ${ }^{100}$ A. Kamenev, ${ }^{100}$ V. Karjavine, ${ }^{100}$ A. Lanev, ${ }^{100}$ A. Malakhov, ${ }^{100}$ V. Matveev, ${ }^{100, m m, n n}$ P. Moisenz,${ }^{100}$ V. Palichik,${ }^{100}$ V. Perelygin, ${ }^{100}$ S. Shmatov ${ }^{100}$ S. Shulha, ${ }^{100}$ N. Skatchkov, ${ }^{100}$ V. Smirnov, ${ }^{100}$ N. Voytishin, ${ }^{100}$ A. Zarubin, ${ }^{100}$ V. Golovtsov, ${ }^{101}$ Y. Ivanov, ${ }^{101}$ V. Kim, ${ }^{101, o o}$ E. Kuznetsova, ${ }^{101, p p}$ P. Levchenko, ${ }^{101}$ V. Murzin, ${ }^{101}$ V. Oreshkin, ${ }^{101}$ I. Smirnov, ${ }^{101}$ D. Sosnov, ${ }^{101}$ V. Sulimov, ${ }^{101}$ L. Uvarov, ${ }^{101}$ S. Vavilov, ${ }^{101}$ A. Vorobyev, ${ }^{101}$ Yu. Andreev, ${ }^{102}$ A. Dermenev, ${ }^{102}$ S. Gninenko, ${ }^{102}$ N. Golubev, ${ }^{102}$ A. Karneyeu, ${ }^{102}$ M. Kirsanov, ${ }^{102}$ N. Krasnikov, ${ }^{102}$ A. Pashenkov, ${ }^{102}$ D. Tlisov,${ }^{102}$ A. Toropin, ${ }^{102}$ V. Epshteyn, ${ }^{103}$ V. Gavrilov, ${ }^{103}$ N. Lychkovskaya, ${ }^{103}$ V. Popov, ${ }^{103}$ I. Pozdnyakov, ${ }^{103}$ G. Safronov, ${ }^{103}$ A. Spiridonov, ${ }^{103}$ A. Stepennov, ${ }^{103}$ V. Stolin, ${ }^{103}$ M. Toms, ${ }^{103}$ E. Vlasov, ${ }^{103}$ A. Zhokin, ${ }^{103}$ T. Aushev,${ }^{104}$ R. Chistov, ${ }^{105, q q}$ M. Danilov, ${ }^{105, q q}$ P. Parygin, ${ }^{105}$ D. Philippov, ${ }^{105}$ S. Polikarpov, ${ }^{105, q q}$ E. Tarkovskii, ${ }^{105}$ V. Andreev, ${ }^{106}$ M. Azarkin, ${ }^{106}$ I. Dremin, ${ }^{106, n n}$ M. Kirakosyan, ${ }^{106}$ A. Terkulov, ${ }^{106}$ A. Baskakov, ${ }^{107}$ A. Belyaev, ${ }^{107}$ E. Boos,${ }^{107}$ M. Dubinin, ${ }^{107, r r}$ L. Dudko, ${ }^{107}$ A. Ershov ${ }^{107}$ A. Gribushin, ${ }^{107}$ V. Klyukhin, ${ }^{107}$ O. Kodolova, ${ }^{107}$ I. Lokhtin, ${ }^{107}$ I. Miagkov, ${ }^{107}$ S. Obraztsov, ${ }^{107}$ S. Petrushanko, ${ }^{107}$ V. Savrin, ${ }^{107}$ A. Snigirev, ${ }^{107}$ A. Barnyakov, ${ }^{108, s s}$ V. Blinov, ${ }^{108, s s}$ T. Dimova, ${ }^{108, s s}$ L. Kardapoltsev, ${ }^{108, s s}$ Y. Skovpen, ${ }^{108, s s}$ I. Azhgirey, ${ }^{109}$ I. Bayshev, ${ }^{109}$ S. Bitioukov, ${ }^{109}$ V. Kachanov, ${ }^{109}$ A. Kalinin, ${ }^{109}$ D. Konstantinov, ${ }^{109}$ P. Mandrik, ${ }^{109}$ V. Petrov ${ }^{109}$ R. Ryutin, ${ }^{109}$ S. Slabospitskii, ${ }^{109}$ A. Sobol, ${ }^{109}$ S. Troshin, ${ }^{109}$ N. Tyurin, ${ }^{109}$ A. Uzunian, ${ }^{109}$ A. Volkov, ${ }^{109}$ A. Babaev, ${ }^{10}$ S. Baidali, ${ }^{110}$ V. Okhotnikov, ${ }^{110}$ P. Adzic, ${ }^{11, t t}$ P. Cirkovic, ${ }^{111}$ D. Devetak, ${ }^{111}$ M. Dordevic, ${ }^{111}$ J. Milosevic, ${ }^{111}$ J. Alcaraz Maestre, ${ }^{112}$ A. Álvarez Fernández, ${ }^{112}$ I. Bachiller, ${ }^{112}$ M. Barrio Luna, ${ }^{112}$ J. A. Brochero Cifuentes, ${ }^{112}$ M. Cerrada,${ }^{112}$ N. Colino, ${ }^{112}$ B. De La Cruz, ${ }^{112}$ A. Delgado Peris, ${ }^{112}$ C. Fernandez Bedoya, ${ }^{112}$ J. P. Fernández Ramos, ${ }^{112}$ J. Flix, ${ }^{112}$ M. C. Fouz, ${ }^{112}$ O. Gonzalez Lopez, ${ }^{112}$ S. Goy Lopez, ${ }^{112}$ J. M. Hernandez, ${ }^{112}$ M. I. Josa ${ }_{112}^{11}$ D. Moran, ${ }^{112}$ A. Pérez-Calero Yzquierdo, ${ }^{112}$ J. Puerta Pelayo, ${ }^{112}$ I. Redondo, ${ }^{112}$ L. Romero, ${ }^{112}$ S. Sánchez Navas, ${ }^{112}$ M. S. Soares, ${ }^{112}$ A. Triossi, ${ }^{112}$ C. Albajar, ${ }^{113}$ J. F. de Trocóniz, ${ }^{113}$ J. Cuevas, ${ }^{114}$ C. Erice, ${ }^{114}$ J. Fernandez Menendez, ${ }^{114}$ S. Folgueras, ${ }^{114}$ I. Gonzalez Caballero, ${ }^{114}$ J. R. González Fernández, ${ }^{114}$ E. Palencia Cortezon, ${ }^{114}$ V. Rodríguez Bouza ${ }^{114}$ S. Sanchez Cruz, ${ }^{114}$ J. M. Vizan Garcia, ${ }^{114}$ I. J. Cabrillo, ${ }^{115}$ A. Calderon, ${ }^{115}$ B. Chazin Quero, ${ }^{115}$ J. Duarte Campderros, ${ }^{115}$ M. Fernandez,${ }^{115}$ P. J. Fernández Manteca, ${ }^{115}$ A. García Alonso, ${ }^{115}$ J. Garcia-Ferrero, ${ }^{115}$ G. Gomez, ${ }^{115}$ A. Lopez Virto, ${ }^{115}$ J. Marco, ${ }^{115}$ C. Martinez Rivero, ${ }^{115}$ P. Martinez Ruiz del Arbol, ${ }^{115}$ F. Matorras, ${ }^{115}$ J. Piedra Gomez, ${ }^{115}$ C. Prieels, ${ }^{115}$ T. Rodrigo, ${ }^{115}$ A. Ruiz-Jimeno, ${ }^{115}$ L. Scodellaro, ${ }^{115}$ N. Trevisani ${ }_{1}^{115}$ I. Vila, ${ }^{115}$ R. Vilar Cortabitarte, ${ }^{115}$ N. Wickramage, ${ }^{116}$ D. Abbaneo,${ }^{117}$ B. Akgun, ${ }^{117}$ E. Auffray, ${ }^{117}$ G. Auzinger, ${ }^{117}$ P. Baillon, ${ }^{117}$ A. H. Ball, ${ }^{117}$ D. Barney, ${ }^{117}$ J. Bendavid, ${ }^{117}$ M. Bianco, ${ }^{117}$ A. Bocci, ${ }^{117}$ C. Botta, ${ }^{117}$ E. Brondolin, ${ }^{117}$ T. Camporesi,${ }^{117}$ M. Cepeda, ${ }^{117}$ G. Cerminara, ${ }^{117}$ E. Chapon, ${ }^{117}$ Y. Chen,,${ }^{117}$ G. Cucciati, ${ }^{117}$ D. d'Enterria, ${ }^{117}$ A. Dabrowski, ${ }^{117}$ N. Daci, ${ }^{117}$ V. Daponte, ${ }^{117}$ A. David, ${ }^{117}$ A. De Roeck, ${ }^{117}$ N. Deelen, ${ }^{117}$ M. Dobson, ${ }^{117}$ M. Dünser, ${ }^{117}$ N. Dupont, ${ }^{117}$ A. Elliott-Peisert, ${ }^{117}$ P. Everaerts, ${ }^{117}$ F. Fallavollita, ${ }^{117, \text { uu }}$ D. Fasanella, ${ }^{117}$ G. Franzoni, ${ }^{117}$ J. Fulcher, ${ }^{117}$ W. Funk, ${ }^{117}$ D. Gigi,${ }^{117}$ A. Gilbert, ${ }^{117}$ K. Gill, ${ }^{117}$ F. Glege, ${ }^{117}$ M. Gruchala,${ }^{117}$ M. Guilbaud,${ }^{117}$ D. Gulhan, ${ }^{117}$ J. Hegeman, ${ }^{117}$ C. Heidegger, ${ }^{117}$ V. Innocente, ${ }^{117}$ A. Jafari, ${ }^{117}$ P. Janot, ${ }^{117}$ O. Karacheban, ${ }^{117, u}$ J. Kieseler, ${ }^{117}$ A. Kornmayer, ${ }^{117}$ M. Krammer, ${ }^{117, b}$ C. Lange, ${ }^{117}$ P. Lecoq,${ }^{117}$ C. Lourenço, ${ }^{117}$ L. Malgeri, ${ }^{117}$ M. Mannelli, ${ }^{117}$ A. Massironi, ${ }^{117}$ F. Meijers, ${ }^{117}$ J. A. Merlin, ${ }^{117}$ S. Mersi,${ }^{117}$ E. Meschi, ${ }^{117}$ P. Milenovic, ${ }^{117, v v}$ F. Moortgat,${ }^{117}$ M. Mulders, ${ }^{117}$ J. Ngadiuba, ${ }^{117}$ S. Nourbakhsh, ${ }^{117}$ S. Orfanelli, ${ }^{117}$ L. Orsini, ${ }^{117}$ F. Pantaleo, ${ }^{117, r}$ L. Pape, ${ }^{117}$ E. Perez, ${ }^{117}$ M. Peruzzi, ${ }^{117}$ A. Petrilli, ${ }^{117}$ G. Petrucciani, ${ }^{117}$ A. Pfeiffer, ${ }^{117}$ M. Pierini, ${ }^{117}$ F. M. Pitters, ${ }^{117}$ D. Rabady, ${ }^{117}$ A. Racz, ${ }^{117}$ T. Reis, ${ }^{117}$ M. Rovere, ${ }^{117}$ H. Sakulin, ${ }^{117}$ C. Schäfer,${ }^{117}$ C. Schwick, ${ }^{117}$ M. Selvaggi, ${ }^{117}$ A. Sharma,${ }^{117}$ P. Silva, ${ }^{117}$ P. Sphicas, ${ }^{117, w w}$ A. Stakia, ${ }^{117}$ J. Steggemann, ${ }^{117}$ D. Treille, ${ }^{117}$ A. Tsirou, ${ }^{117}$ V. Veckalns, ${ }^{117, x x}$ M. Verzetti, ${ }^{117}$ W. D. Zeuner, ${ }^{117}$ L. Caminada, ${ }^{118, y y}$ K. Deiters,${ }^{118}$ W. Erdmann, ${ }^{118}$ R. Horisberger, ${ }^{118}$ Q. Ingram,${ }^{118}$ H. C. Kaestli, ${ }^{118}$ D. Kotlinski, ${ }^{118}$ U. Langenegger, ${ }^{118}$ T. Rohe, ${ }^{118}$ S. A. Wiederkehr, ${ }^{118}$ M. Backhaus, ${ }^{119}$ L. Bäni, ${ }^{119}$ P. Berger, ${ }^{119}$ N. Chernyavskaya, ${ }^{119}$ G. Dissertori, ${ }^{119}$ M. Dittmar, ${ }^{119}$ M. Donegà,${ }^{119}$ C. Dorfer,${ }^{119}$ T. A. Gómez Espinosa, ${ }^{119}$ C. Grab,${ }^{119}$ D. Hits, ${ }^{119}$ T. Klijnsma, ${ }^{119}$ W. Lustermann, ${ }^{119}$ R. A. Manzoni, ${ }^{119}$ M. Marionneau, ${ }^{119}$ M. T. Meinhard, ${ }^{119}$ F. Micheli, ${ }^{119}$ P. Musella, ${ }^{119}$ F. Nessi-Tedaldi, ${ }^{119}$ F. Pauss, ${ }^{119}$ G. Perrin, ${ }^{119}$ L. Perrozzi, ${ }^{119}$ S. Pigazzini, ${ }^{119}$ M. Quittnat, ${ }^{119}$ C. Reissel, ${ }^{119}$ D. Ruini, ${ }^{119}$ 
D. A. Sanz Becerra, ${ }^{119}$ M. Schönenberger, ${ }^{119}$ L. Shchutska, ${ }^{119}$ V. R. Tavolaro, ${ }^{119}$ K. Theofilatos, ${ }^{119}$

M. L. Vesterbacka Olsson, ${ }^{119}$ R. Wallny, ${ }^{119}$ D. H. Zhu, ${ }^{119}$ T. K. Aarrestad, ${ }^{120}$ C. Amsler, ${ }^{120, z z}$ D. Brzhechko, ${ }^{120}$ M. F. Canelli, ${ }^{120}$ A. De Cosa, ${ }^{120}$ R. Del Burgo, ${ }^{120}$ S. Donato, ${ }^{120}$ C. Galloni, ${ }^{120}$ T. Hreus, ${ }^{120}$ B. Kilminster, ${ }^{120}$ S. Leontsinis, ${ }^{120}$ I. Neutelings, ${ }^{120}$ G. Rauco, ${ }^{120}$ P. Robmann, ${ }^{120}$ K. Schweiger, ${ }^{120}$ C. Seitz,${ }^{120}$ Y. Takahashi, ${ }^{120}$ A. Zucchetta, ${ }^{120}$ T. H. Doan, ${ }^{121}$ R. Khurana, ${ }^{121}$ C. M. Kuo, ${ }^{121}$ W. Lin, ${ }^{121}$ A. Pozdnyakov, ${ }^{121}$ S. S. Yu, ${ }^{121}$ P. Chang, ${ }^{122}$ Y. Chao, ${ }^{122}$ K. F. Chen, ${ }^{122}$ P. H. Chen, ${ }^{122}$ W.-S. Hou, ${ }^{122}$ Y. F. Liu, ${ }^{122}$ R.-S. Lu, ${ }^{122}$ E. Paganis, ${ }^{122}$ A. Psallidas, ${ }^{122}$ A. Steen, ${ }^{122}$ B. Asavapibhop, ${ }^{123}$ N. Srimanobhas, ${ }^{123}$ N. Suwonjandee, ${ }^{123}$ A. Bat, ${ }^{124}$ F. Boran, ${ }^{124}$ S. Cerci, ${ }^{124, \text { aaa }}$ S. Damarseckin, ${ }^{124}$ Z. S. Demiroglu, ${ }^{124}$ F. Dolek, ${ }^{124}$ C. Dozen, ${ }^{124}$ I. Dumanoglu, ${ }^{124}$ G. Gokbulut, ${ }^{124}$ Y. Guler, ${ }^{124}$ E. Gurpinar, ${ }^{124}$ I. Hos, ${ }^{124, b b b}$ C. Isik, ${ }^{124}$ E. E. Kangal, ${ }^{124, c c c}$ O. Kara, ${ }^{124}$ A. Kayis Topaksu, ${ }^{124}$ U. Kiminsu, ${ }^{124}$ M. Oglakci, ${ }^{124}$ G. Onengut, ${ }^{124}$ K. Ozdemir, ${ }^{124, d d d}$ S. Ozturk, ${ }^{124, \text { eee }}$ D. Sunar Cerci, ${ }^{124, \text { aaa }}$ B. Tali, ${ }^{124, \text { aaa }}$ U. G. Tok, ${ }^{124}$ S. Turkcapar, ${ }^{124}$ I. S. Zorbakir, ${ }^{124}$ C. Zorbilmez, ${ }^{124}$ B. Isildak, ${ }^{125, \text { fff }}$ G. Karapinar, ${ }^{125, g g g}$ M. Yalvac, ${ }^{125}$ M. Zeyrek, ${ }^{125}$ I. O. Atakisi, ${ }^{126}$ E. Gülmez, ${ }^{126}$ M. Kaya, ${ }^{126, \text { hhh }}$ O. Kaya, ${ }^{126, \text { iii }}$ S. Ozkorucuklu, ${ }^{126, j j i}$ S. Tekten, ${ }^{126}$ E. A. Yetkin, ${ }^{126, k k k}$ M. N. Agaras, ${ }^{127}$ A. Cakir, ${ }^{127}$ K. Cankocak, ${ }^{127}$ Y. Komurcu, ${ }^{127}$ S. Sen, ${ }^{127,111}$ B. Grynyov, ${ }^{128}$ L. Levchuk, ${ }^{129}$ F. Ball, ${ }^{130}$ J. J. Brooke, ${ }^{130}$ D. Burns, ${ }^{130}$ E. Clement, ${ }^{130}$ D. Cussans, ${ }^{130}$ O. Davignon, ${ }^{130}$ H. Flacher, ${ }^{130}$ J. Goldstein, ${ }^{130}$ G. P. Heath,${ }^{130}$ H. F. Heath,${ }^{130}$ L. Kreczko, ${ }^{130}$ D. M. Newbold, ${ }^{130, m m m}$ S. Paramesvaran, ${ }^{130}$ B. Penning, ${ }^{130}$ T. Sakuma, ${ }^{130}$ D. Smith, ${ }^{130}$ V. J. Smith, ${ }^{130}$ J. Taylor, ${ }^{130}$ A. Titterton, ${ }^{130}$ K. W. Bell, ${ }^{131}$ A. Belyaev, ${ }^{131, n n n}$ C. Brew, ${ }^{131}$ R. M. Brown, ${ }^{131}$ D. Cieri, ${ }^{131}$ D. J. A. Cockerill, ${ }^{131}$ J. A. Coughlan, ${ }^{131}$ K. Harder, ${ }^{131}$ S. Harper, ${ }^{131}$ J. Linacre, ${ }^{131}$ K. Manolopoulos, ${ }^{131}$ E. Olaiyaa ${ }^{131}$ D. Petyt ${ }^{131}$ C. H. Shepherd-Themistocleous, ${ }^{131}$ A. Thea, ${ }^{131}$ I. R. Tomalin, ${ }^{131}$ T. Williams, ${ }^{131}$ W. J. Womersley, ${ }^{131}$ R. Bainbridge, ${ }^{132}$ P. Bloch, ${ }^{132}$ J. Borg, ${ }^{132}$ S. Breeze, ${ }^{132}$ O. Buchmuller, ${ }^{132}$ A. Bundock, ${ }^{132}$ D. Colling, ${ }^{132}$ P. Dauncey, ${ }^{132}$ G. Davies,${ }^{132}$ M. Della Negra, ${ }^{132}$ R. Di Maria, ${ }^{132}$ G. Hall, ${ }^{132}$ G. Iles, ${ }^{132}$ T. James, ${ }^{132}$ M. Komm, ${ }^{132}$ L. Lyons, ${ }^{132}$ A.-M. Magnan, ${ }^{132}$ S. Malik,${ }^{132}$ A. Martelli, ${ }^{132}$ J. Nash, ${ }^{132,000}$ A. Nikitenko, ${ }^{132, \mathrm{~h}}$ V. Palladino, ${ }^{132}$ M. Pesaresi, ${ }^{132}$ D. M. Raymond, ${ }^{132}$ A. Richards, ${ }^{132}$ A. Rose, ${ }^{132}$ E. Scott, ${ }^{132}$ C. Seez, ${ }^{132}$ A. Shtipliyski, ${ }^{132}$ G. Singh, ${ }^{132}$ M. Stoye, ${ }^{132}$ T. Strebler, ${ }^{132}$ S. Summers, ${ }^{132}$ A. Tapper,${ }^{132}$ K. Uchida,${ }^{132}$ T. Virdee, ${ }^{132, r}$ N. Wardle, ${ }^{132}$ D. Winterbottom, ${ }^{132}$ S. C. Zenz, ${ }^{132}$ J. E. Cole, ${ }^{133}$ P. R. Hobson, ${ }^{133}$ A. Khan,${ }^{133}$ P. Kyberd, ${ }^{133}$ C. K. Mackay, ${ }^{133}$

A. Morton, ${ }^{133}$ I. D. Reid, ${ }^{133}$ L. Teodorescu, ${ }^{133}$ S. Zahid, ${ }^{133}$ K. Call, ${ }^{134}$ J. Dittmann, ${ }^{134}$ K. Hatakeyama, ${ }^{134}$ H. Liu, ${ }^{134}$ C. Madrid, ${ }^{134}$ B. McMaster, ${ }^{134}$ N. Pastika ${ }^{134}$ C. Smith, ${ }^{134}$ R. Bartek, ${ }^{135}$ A. Dominguez, ${ }^{135}$ A. Buccilli, ${ }^{136}$ S. I. Cooper, ${ }^{136}$ C. Henderson, ${ }^{136}$ P. Rumerio, ${ }^{136}$ C. West, ${ }^{136}$ D. Arcaro, ${ }^{137}$ T. Bose, ${ }^{137}$ D. Gastler, ${ }^{137}$ S. Girgis,${ }^{137}$ D. Pinna, ${ }^{137}$ D. Rankin, ${ }^{137}$ C. Richardson, ${ }^{137}$ J. Rohlf,${ }^{137}$ L. Sulak, ${ }^{137}$ D. Zou, ${ }^{137}$ G. Benelli, ${ }^{138}$ X. Coubez, ${ }^{138}$ D. Cutts, ${ }^{138}$ M. Hadley, ${ }^{138}$ J. Hakala, ${ }^{138}$ U. Heintz, ${ }^{138}$ J. M. Hogan, ${ }^{138, p p p}$ K. H. M. Kwok, ${ }^{138}$ E. Laird, ${ }^{138}$ G. Landsberg, ${ }^{138}$ J. Lee, ${ }^{138}$ Z. Mao, ${ }^{138}$ M. Narain, ${ }^{138}$ S. Sagir, ${ }^{138, q q 9}$ R. Syarif, ${ }^{138}$ E. Usai, ${ }^{138}$ D. Yu, ${ }^{138}$ R. Band, ${ }^{139}$ C. Brainerd, ${ }^{139}$ R. Breedon, ${ }^{139}$ D. Burns, ${ }^{139}$ M. Calderon De La Barca Sanchez, ${ }^{139}$ M. Chertok, ${ }^{139}$ J. Conway, ${ }^{139}$ R. Conway, ${ }^{139}$ P. T. Cox, ${ }^{139}$ R. Erbacher, ${ }^{139}$ C. Flores, ${ }^{139}$ G. Funk, ${ }^{139}$ W. Ko, ${ }^{139}$ O. Kukral, ${ }^{139}$ R. Lander, ${ }^{139}$ M. Mulhearn, ${ }^{139}$ D. Pellett, ${ }^{139}$ J. Pilot, ${ }^{139}$ S. Shalhout, ${ }^{139}$ M. Shi, ${ }^{139}$ D. Stolp, ${ }^{139}$ D. Taylor, ${ }^{139}$ K. Tos, ${ }^{139}$ M. Tripathi, ${ }^{139}$ Z. Wang, ${ }^{139}$ F. Zhang, ${ }^{139}$ M. Bachtis, ${ }^{140}$ C. Bravo, ${ }^{140}$ R. Cousins, ${ }^{140}$ A. Dasgupta, ${ }^{140}$ A. Florent, ${ }^{140}$ J. Hauser,${ }^{140}$ M. Ignatenko, ${ }^{140}$ N. Mccoll, ${ }^{140}$ S. Regnard, ${ }^{140}$ D. Saltzberg, ${ }^{140}$ C. Schnaible, ${ }^{140}$ V. Valuev, ${ }^{140}$ E. Bouvier, ${ }^{141}$ K. Burt, ${ }^{141}$ R. Clare,${ }^{141}$ J. W. Gary, ${ }^{141}$ S. M. A. Ghiasi Shirazi, ${ }^{141}$ G. Hanson, ${ }^{141}$ G. Karapostoli, ${ }^{141}$ E. Kennedy, ${ }^{141}$ F. Lacroix, ${ }^{141}$ O. R. Long, ${ }^{141}$ M. Olmedo Negrete, ${ }^{141}$ M. I. Paneva, ${ }^{141}$ W. Si, ${ }^{141}$ L. Wang, ${ }^{141}$ H. Wei, ${ }^{141}$ S. Wimpenny, ${ }^{141}$ B. R. Yates, ${ }^{141}$ J. G. Branson, ${ }^{142}$ P. Chang, ${ }^{142}$ S. Cittolin, ${ }^{142}$ M. Derdzinski, ${ }^{142}$ R. Gerosa, ${ }^{142}$ D. Gilbert, ${ }^{142}$ B. Hashemi, ${ }^{142}$ A. Holzner, ${ }^{142}$ D. Klein, ${ }^{142}$ G. Kole, ${ }^{142}$ V. Krutelyov, ${ }^{142}$ J. Letts, ${ }^{142}$ M. Masciovecchio, ${ }^{142}$ D. Olivito, ${ }^{142}$ S. Padhi, ${ }^{142}$ M. Pieri, ${ }^{142}$ M. Sani, ${ }^{142}$ V. Sharma, ${ }^{142}$ S. Simon, ${ }^{142}$ M. Tadel,${ }^{142}$ A. Vartak, ${ }^{142}$ S. Wasserbaech, ${ }^{142, \text { rrr }}$ J. Wood, ${ }^{142}$ F. Würthwein, ${ }^{142}$ A. Yagil, ${ }^{142}$ G. Zevi Della Porta, ${ }^{142}$ N. Amin, ${ }^{143}$ R. Bhandari, ${ }^{143}$ C. Campagnari, ${ }^{143}$ M. Citron, ${ }^{143}$ V. Dutta, ${ }^{143}$ M. Franco Sevilla, ${ }^{143}$ L. Gouskos, ${ }^{143}$ R. Heller, ${ }^{143}$ J. Incandela, ${ }^{143}$ H. Mei, ${ }^{143}$ A. Ovcharova, ${ }^{143}$ H. Qu, ${ }^{143}$ J. Richman, ${ }^{143}$ D. Stuart, ${ }^{143}$ I. Suarez, ${ }^{143}$ S. Wang, ${ }^{143}$ J. Yoo, ${ }^{143}$ D. Anderson, ${ }^{144}$ A. Bornheim, ${ }^{144}$ J. M. Lawhorn, ${ }^{144}$ N. Lu, ${ }^{144}$ H. B. Newman, ${ }^{144}$ T. Q. Nguyen, ${ }^{144}$ J. Pata, ${ }^{144}$ M. Spiropulu, ${ }^{144}$ J. R. Vlimant, ${ }^{144}$ R. Wilkinson, ${ }^{144}$ S. Xie, ${ }^{144}$ Z. Zhang, ${ }^{144}$ R. Y. Zhu, ${ }^{144}$ M. B. Andrews, ${ }^{145}$ T. Ferguson, ${ }^{145}$ T. Mudholkar, ${ }^{145}$ M. Paulini, ${ }^{145}$ M. Sun, ${ }^{145}$ I. Vorobiev, ${ }^{145}$ M. Weinberg, ${ }^{145}$ J. P. Cumalat, ${ }^{146}$ W. T. Ford, ${ }^{146}$ F. Jensen, ${ }^{146}$ A. Johnson, ${ }^{146}$ E. MacDonald, ${ }^{146}$ T. Mulholland, ${ }^{146}$ R. Patel,${ }^{146}$ A. Perloff, ${ }^{146}$ K. Stenson, ${ }^{146}$ K. A. Ulmer, ${ }^{146}$

S. R. Wagner, ${ }^{146}$ J. Alexander, ${ }^{147}$ J. Chaves, ${ }^{147}$ Y. Cheng, ${ }^{147}$ J. Chu, ${ }^{147}$ A. Datta, ${ }^{147}$ K. Mcdermott, ${ }^{147}$ N. Mirman, ${ }^{147}$ J. R. Patterson, ${ }^{147}$ D. Quach,${ }^{147}$ A. Rinkevicius, ${ }^{147}$ A. Ryd ${ }^{147}$ L. Skinnari, ${ }^{147}$ L. Soffi, ${ }^{147}$ S. M. Tan,${ }^{147}$ Z. Tao, ${ }^{147}$ J. Thom, ${ }^{147}$ J. Tucker, ${ }^{147}$ P. Wittich, ${ }^{147}$ M. Zientek, ${ }^{147}$ S. Abdullin, ${ }^{148}$ M. Albrow, ${ }^{148}$ M. Alyari, ${ }^{148}$ G. Apollinari, ${ }^{148}$ A. Apresyan, ${ }^{148}$ A. Apyan, ${ }^{148}$ S. Banerjee, ${ }^{148}$ L. A. T. Bauerdick, ${ }^{148}$ A. Beretvas, ${ }^{148}$ J. Berryhill, ${ }^{148}$ P. C. Bhat, ${ }^{148}$ K. Burkett, ${ }^{148}$ 
J. N. Butler, ${ }^{148}$ A. Canepa ${ }^{148}$ G. B. Cerati, ${ }^{148}$ H. W. K. Cheung, ${ }^{148}$ F. Chlebana,${ }^{148}$ M. Cremonesi ${ }^{148}$ J. Duarte, ${ }^{148}$ V. D. Elvira, ${ }^{148}$ J. Freeman, ${ }^{148}$ Z. Gecse, ${ }^{148}$ E. Gottschalk, ${ }^{148}$ L. Gray, ${ }^{148}$ D. Green, ${ }^{148}$ S. Grünendahl, ${ }^{148}$ O. Gutsche, ${ }^{148}$ J. Hanlon, ${ }^{148}$ R. M. Harris, ${ }^{148}$ S. Hasegawa, ${ }^{148}$ J. Hirschauer, ${ }^{148}$ Z. Hu, ${ }^{148}$ B. Jayatilaka, ${ }^{148}$ S. Jindariani, ${ }^{148}$ M. Johnson, ${ }^{148}$ U. Joshi, ${ }^{148}$ B. Klima, ${ }^{148}$ M. J. Kortelainen, ${ }^{148}$ B. Kreis, ${ }^{148}$ S. Lammel, ${ }^{148}$ D. Lincoln, ${ }^{148}$ R. Lipton, ${ }^{148}$ M. Liu, ${ }^{148}$ T. Liu, ${ }^{148}$ J. Lykken, ${ }^{148}$ K. Maeshima, ${ }^{148}$ J. M. Marraffino, ${ }^{148}$ D. Mason, ${ }^{148}$ P. McBride,${ }^{148}$ P. Merkel, ${ }^{148}$ S. Mrenna, ${ }^{148}$ S. Nahn, ${ }^{148}$ V. O’Dell, ${ }^{148}$ K. Pedro, ${ }^{148}$ C. Pena,${ }^{148}$ O. Prokofyev,${ }^{148}$ G. Rakness, ${ }^{148}$ L. Ristori, ${ }^{148}$ A. Savoy-Navarro, ${ }^{148, s s s}$ B. Schneider, ${ }^{148}$ E. Sexton-Kennedy, ${ }^{148}$ A. Soha, ${ }^{148}$ W. J. Spalding, ${ }^{148}$ L. Spiegel, ${ }^{148}$ S. Stoynev, ${ }^{148}$ J. Strait, ${ }^{148}$ N. Strobbe,${ }^{148}$ L. Taylor, ${ }^{148}$ S. Tkaczyk,${ }^{148}$ N. V. Tran, ${ }^{148}$ L. Uplegger,${ }^{148}$ E. W. Vaandering, ${ }^{148}$ C. Vernieri, ${ }^{148}$ M. Verzocchi, ${ }^{148}$ R. Vidal, ${ }^{148}$ M. Wang, ${ }^{148}$ H. A. Weber, ${ }^{148}$ A. Whitbeck, ${ }^{148}$ D. Acosta, ${ }^{149}$ P. Avery, ${ }^{149}$ P. Bortignon, ${ }^{149}$ D. Bourilkov, ${ }^{149}$ A. Brinkerhoff, ${ }^{149}$

L. Cadamuro, ${ }^{149}$ A. Carnes, ${ }^{149}$ D. Curry ${ }^{149}$ R. D. Field, ${ }^{149}$ S. V. Gleyzer, ${ }^{149}$ B. M. Joshi, ${ }^{149}$ J. Konigsberg, ${ }^{149}$ A. Korytov, ${ }^{149}$ K. H. Lo, ${ }^{149}$ P. Ma, ${ }^{149}$ K. Matchev, ${ }^{149}$ G. Mitselmakher, ${ }^{149}$ D. Rosenzweig, ${ }^{149}$ K. Shi, ${ }^{149}$ D. Sperka, ${ }^{149}$ J. Wang, ${ }^{149}$ S. Wang, ${ }^{149}$ X. Zuo ${ }^{149}$ Y. R. Joshi,${ }^{150}$ S. Linn, ${ }^{150}$ A. Ackert, ${ }^{151}$ T. Adams, ${ }^{151}$ A. Askew, ${ }^{151}$ S. Hagopian, ${ }^{151}$ V. Hagopian, ${ }^{151}$ K. F. Johnson, ${ }^{151}$ T. Kolberg, ${ }^{151}$ G. Martinez, ${ }^{151}$ T. Perry, ${ }^{151}$ H. Prosper ${ }^{151}$ A. Saha, ${ }^{151}$ C. Schiber, ${ }^{151}$ R. Yohay, ${ }^{151}$ M. M. Baarmand, ${ }^{152}$ V. Bhopatkar, ${ }^{152}$ S. Colafranceschi, ${ }^{152}$ M. Hohlmann, ${ }^{152}$ D. Noonan, ${ }^{152}$ M. Rahmani, ${ }^{152}$ T. Roy, ${ }^{152}$ F. Yumiceva, ${ }^{152}$ M. R. Adams, ${ }^{153}$ L. Apanasevich, ${ }^{153}$ D. Berry, ${ }^{153}$ R. R. Betts, ${ }^{153}$ R. Cavanaugh, ${ }^{153}$ X. Chen, ${ }^{153}$ S. Dittmer, ${ }^{153}$ O. Evdokimov, ${ }^{153}$ C. E. Gerber, ${ }^{153}$ D. A. Hangal, ${ }^{153}$ D. J. Hofman, ${ }^{153}$ K. Jung, ${ }^{153}$ J. Kamin,${ }^{153}$ C. Mills, ${ }^{153}$ M. B. Tonjes, ${ }^{153}$ N. Varelas, ${ }^{153}$ H. Wang,${ }^{153}$ X. Wang, ${ }^{153}$ Z. Wu, ${ }^{153}$ J. Zhang, ${ }^{153}$ M. Alhusseini, ${ }^{154}$ B. Bilki, ${ }^{154, \text { tt }}$ W. Clarida,${ }^{154}$ K. Dilsiz, ${ }^{154, \text { uuu }}$ S. Durgut, ${ }^{154}$ R. P. Gandrajula, ${ }^{154}$ M. Haytmyradov, ${ }^{154}$ V. Khristenko, ${ }^{154}$ J.-P. Merlo, ${ }^{154}$ A. Mestvirishvili, ${ }^{154}$ A. Moeller, ${ }^{154}$

J. Nachtman, ${ }^{154}$ H. Ogul, ${ }^{154, v v v}$ Y. Onel, ${ }^{154}$ F. Ozok, ${ }^{154, w w w}$ A. Penzo, ${ }^{154}$ C. Snyder,${ }^{154}$ E. Tiras, ${ }^{154}$ J. Wetzel,${ }^{154}$

B. Blumenfeld, ${ }^{155}$ A. Cocoros, ${ }^{155}$ N. Eminizer, ${ }^{155}$ D. Fehling, ${ }^{155}$ L. Feng, ${ }^{155}$ A. V. Gritsan, ${ }^{155}$ W. T. Hung, ${ }^{155}$

P. Maksimovic, ${ }^{155}$ J. Roskes, ${ }^{155}$ U. Sarica, ${ }^{155}$ M. Swartz, ${ }^{155}$ M. Xiao, ${ }^{155}$ C. You, ${ }^{155}$ A. Al-bataineh, ${ }^{156}$ P. Baringer, ${ }^{156}$ A. Bean, ${ }^{156}$ S. Boren, ${ }^{156}$ J. Bowen, ${ }^{156}$ A. Bylinkin, ${ }^{156}$ J. Castle, ${ }^{156}$ S. Khalil, ${ }^{156}$ A. Kropivnitskaya, ${ }^{156}$ D. Majumder, ${ }^{156}$ W. Mcbrayer, ${ }^{156}$ M. Murray, ${ }^{156}$ C. Rogan, ${ }^{156}$ S. Sanders, ${ }^{156}$ E. Schmitz, ${ }^{156}$ J. D. Tapia Takaki, ${ }^{156}$ Q. Wang, ${ }^{156}$ S. Duric, ${ }^{157}$ A. Ivanov, ${ }^{157}$ K. Kaadze, ${ }^{157}$ D. Kim, ${ }^{157}$ Y. Maravin, ${ }^{157}$ D. R. Mendis, ${ }^{157}$ T. Mitchell, ${ }^{157}$ A. Modak, ${ }^{157}$ A. Mohammadi, ${ }^{157}$ F. Rebassoo, ${ }^{158}$ D. Wright, ${ }^{158}$ A. Baden, ${ }^{159}$ O. Baron, ${ }^{159}$ A. Belloni, ${ }^{159}$ S. C. Eno, ${ }^{159}$ Y. Feng, ${ }^{159}$ C. Ferraioli, ${ }^{159}$ N. J. Hadley, ${ }^{159}$ S. Jabeen, ${ }^{159}$ G. Y. Jeng, ${ }^{159}$ R. G. Kellogg, ${ }^{159}$ J. Kunkle, ${ }^{159}$ A. C. Mignerey, ${ }^{159}$ S. Nabili, ${ }^{159}$ F. Ricci-Tam, ${ }^{159}$ M. Seidel, ${ }^{159}$ Y. H. Shin, ${ }^{159}$ A. Skuja, ${ }^{159}$ S. C. Tonwar, ${ }^{159}$ K. Wong, ${ }^{159}$ D. Abercrombie, ${ }^{160}$ B. Allen, ${ }^{160}$ V. Azzolini, ${ }^{160}$

A. Baty, ${ }^{160}$ G. Bauer, ${ }^{160}$ R. Bi, ${ }^{160}$ S. Brandt, ${ }^{160}$ W. Busza, ${ }^{160}$ I. A. Cali, ${ }^{160}$ M. D’ Alfonso, ${ }^{160}$ Z. Demiragli, ${ }^{160}$ G. Gomez Ceballos, ${ }^{160}$ M. Goncharov, ${ }^{160}$ P. Harris, ${ }^{160}$ D. Hsu, ${ }^{160}$ M. Hu, ${ }^{160}$ Y. Iiyama, ${ }^{160}$ G. M. Innocenti, ${ }^{160}$ M. Klute, ${ }^{160}$ D. Kovalskyi, ${ }^{160}$ Y.-J. Lee, ${ }^{160}$ P. D. Luckey, ${ }^{160}$ B. Maier ${ }^{160}$ A. C. Marini, ${ }^{160}$ C. Mcginn, ${ }^{160}$ C. Mironov, ${ }^{160}$ S. Narayanan, ${ }^{160}$ X. Niu, ${ }^{160}$ C. Paus, ${ }^{160}$ C. Roland, ${ }^{160}$ G. Roland, ${ }^{160}$ Z. Shi, ${ }^{160}$ G. S. F. Stephans, ${ }^{160}$ K. Sumorok, ${ }^{160}$ K. Tatar, ${ }^{160}$ D. Velicanu, ${ }^{160}$

J. Wang, ${ }^{160}$ T. W. Wang, ${ }^{160}$ B. Wyslouch, ${ }^{160}$ A. C. Benvenuti, ${ }^{161, a}$ R. M. Chatterjee, ${ }^{161}$ A. Evans, ${ }^{161}$ P. Hansen, ${ }^{161}$ J. Hiltbrand, ${ }^{161}$ Sh. Jain, ${ }^{161}$ S. Kalafut, ${ }^{161}$ M. Krohn, ${ }^{161}$ Y. Kubota, ${ }^{161}$ Z. Lesko, ${ }^{161}$ J. Mans, ${ }^{161}$ N. Ruckstuhl,${ }^{161}$ R. Rusack, ${ }^{161}$ M. A. Wadud, ${ }^{161}$ J. G. Acosta, ${ }^{162}$ S. Oliveros, ${ }^{162}$ E. Avdeeva, ${ }^{163}$ K. Bloom,${ }^{163}$ D. R. Claes, ${ }^{163}$ C. Fangmeier, ${ }^{163}$ F. Golf, ${ }^{163}$ R. Gonzalez Suarez, ${ }^{163}$ R. Kamalieddin, ${ }^{163}$ I. Kravchenko, ${ }^{163}$ J. Monroy, ${ }^{163}$ J. E. Siado, ${ }^{163}$ G. R. Snow, ${ }^{163}$ B. Stieger, ${ }^{163}$

A. Godshalk, ${ }^{164}$ C. Harrington, ${ }^{164}$ I. Iashvili, ${ }^{164}$ A. Kharchilava,${ }^{164}$ C. Mclean, ${ }^{164}$ D. Nguyen, ${ }^{164}$ A. Parker, ${ }^{164}$

S. Rappoccio, ${ }^{164}$ B. Roozbahani, ${ }^{164}$ G. Alverson, ${ }^{165}$ E. Barberis, ${ }^{165}$ C. Freer,${ }^{165}$ Y. Haddad, ${ }^{165}$ A. Hortiangtham, ${ }^{165}$

D. M. Morse, ${ }^{165}$ T. Orimoto, ${ }^{165}$ T. Wamorkar, ${ }^{165}$ B. Wang, ${ }^{165}$ A. Wisecarver, ${ }^{165}$ D. Wood, ${ }^{165}$ S. Bhattacharya, ${ }^{166}$

J. Bueghly, ${ }^{166}$ O. Charaf, ${ }^{166}$ T. Gunter, ${ }^{166}$ K. A. Hahn, ${ }^{166}$ N. Odell, ${ }^{166}$ M. H. Schmitt, ${ }^{166}$ K. Sung, ${ }^{166}$ M. Trovato, ${ }^{166}$

M. Velasco, ${ }^{166}$ R. Bucci, ${ }^{167}$ N. Dev ${ }^{167}$ M. Hildreth,${ }^{167}$ K. Hurtado Anampa,${ }^{167}$ C. Jessop, ${ }^{167}$ D. J. Karmgard, ${ }^{167}$

K. Lannon, ${ }^{167}$ W. Li,${ }^{167}$ N. Loukas, ${ }^{167}$ N. Marinelli, ${ }^{167}$ F. Meng, ${ }^{167}$ C. Mueller ${ }^{167}$ Y. Musienko, ${ }^{167, m m}$ M. Planer, ${ }^{167}$ A. Reinsvold, ${ }^{167}$ R. Ruchti, ${ }^{167}$ P. Siddireddy, ${ }^{167}$ G. Smith, ${ }^{167}$ S. Taroni, ${ }^{167}$ M. Wayne, ${ }^{167}$ A. Wightman, ${ }^{167}$ M. Wolf, ${ }^{167}$ A. Woodard, ${ }^{167}$ J. Alimena, ${ }^{168}$ L. Antonelli, ${ }^{168}$ B. Bylsma, ${ }^{168}$ L. S. Durkin, ${ }^{168}$ S. Flowers, ${ }^{168}$ B. Francis, ${ }^{168}$ C. Hill, ${ }^{168}$ W. Ji, ${ }^{168}$ T. Y. Ling, ${ }^{168}$ W. Luo, ${ }^{168}$ B. L. Winer, ${ }^{168}$ S. Cooperstein, ${ }^{169}$ P. Elmer, ${ }^{169}$ J. Hardenbrook, ${ }^{169}$ N. Haubrich, ${ }^{169}$ S. Higginbotham, ${ }^{169}$ A. Kalogeropoulos, ${ }^{169}$ S. Kwan, ${ }^{169}$ D. Lange, ${ }^{169}$ M. T. Lucchini, ${ }^{169}$ J. Luo, ${ }^{169}$ D. Marlow, ${ }^{169}$ K. Mei, ${ }^{169}$ I. Ojalvo, ${ }^{169}$ J. Olsen, ${ }^{169}$ C. Palmer, ${ }^{169}$ P. Piroué, ${ }^{169}$ J. Salfeld-Nebgen, ${ }^{169}$ D. Stickland ${ }^{169}$ C. Tully, ${ }^{169}$ S. Malik, ${ }^{170}$ S. Norberg, ${ }^{170}$ A. Barker, ${ }^{171}$ V. E. Barnes, ${ }^{171}$ S. Das, ${ }^{171}$ L. Gutay, ${ }^{171}$ M. Jones,${ }^{171}$ A. W. Jung, ${ }^{171}$ A. Khatiwada, ${ }^{171}$ B. Mahakud, ${ }^{171}$ D. H. Miller, ${ }^{171}$ N. Neumeister, ${ }^{171}$ C. C. Peng, ${ }^{171}$ S. Piperov,${ }^{171}$ H. Qiu, ${ }^{171}$ J. F. Schulte, ${ }^{171}$ J. Sun, ${ }^{171}$ F. Wang, ${ }^{171}$ R. Xiao, ${ }^{171}$ W. Xie, ${ }^{171}$ T. Cheng, ${ }^{172}$ J. Dolen, ${ }^{172}$ N. Parashar, ${ }^{172}$ Z. Chen, ${ }^{173}$ K. M. Ecklund, ${ }^{173}$ S. Freed, ${ }^{173}$ 
F. J. M. Geurts, ${ }^{173}$ M. Kilpatrick, ${ }^{173}$ Arun Kumar, ${ }^{173}$ W. Li, ${ }^{173}$ B. P. Padley, ${ }^{173}$ R. Redjimi,,${ }^{173}$ J. Roberts,${ }^{173}$ J. Rorie, ${ }^{173}$ W. Shi, ${ }^{173}$ Z. Tu, ${ }^{173}$ A. Zhang, ${ }^{173}$ A. Bodek, ${ }^{174}$ P. de Barbaro, ${ }^{174}$ R. Demina, ${ }^{174}$ Y. t. Duh, ${ }^{174}$ J. L. Dulemba, ${ }^{174}$ C. Fallon, ${ }^{174}$ T. Ferbel, ${ }^{174}$ M. Galanti, ${ }^{174}$ A. Garcia-Bellido, ${ }^{174}$ J. Han,${ }^{174}$ O. Hindrichs, ${ }^{174}$ A. Khukhunaishvili, ${ }^{174}$ E. Ranken, ${ }^{174}$ P. Tan, ${ }^{174}$ R. Taus, ${ }^{174}$ B. Chiarito, ${ }^{175}$ J. P. Chou, ${ }^{175}$ Y. Gershtein, ${ }^{175}$ E. Halkiadakis, ${ }^{175}$ A. Hart, ${ }^{175}$ M. Heindl, ${ }^{175}$ E. Hughes, ${ }^{175}$ S. Kaplan, ${ }^{175}$ R. Kunnawalkam Elayavalli, ${ }^{175}$ S. Kyriacou, ${ }^{175}$ I. Laflotte, ${ }^{175}$ A. Lath, ${ }^{175}$ R. Montalvo, ${ }^{175}$ K. Nash, ${ }^{175}$ M. Osherson, ${ }^{175}$ H. Saka, ${ }^{175}$ S. Salur, ${ }^{175}$ S. Schnetzer, ${ }^{175}$ D. Sheffield, ${ }^{175}$ S. Somalwar, ${ }^{175}$ R. Stone, ${ }^{175}$ S. Thomas, ${ }^{175}$ P. Thomassen, ${ }^{175}$ A. G. Delannoy, ${ }^{176}$ J. Heideman, ${ }^{176}$ G. Riley, ${ }^{176}$ S. Spanier, ${ }^{176}$ O. Bouhali, ${ }^{177, x x x}$ A. Celik, ${ }^{177}$

M. Dalchenko, ${ }^{177}$ M. De Mattia, ${ }^{177}$ A. Delgado, ${ }^{177}$ S. Dildick, ${ }^{177}$ R. Eusebi, ${ }^{177}$ J. Gilmore, ${ }^{177}$ T. Huang, ${ }^{177}$ T. Kamon, ${ }^{177, y y y}$ S. Luo, ${ }^{177}$ D. Marley, ${ }^{177}$ R. Mueller, ${ }^{177}$ D. Overton, ${ }^{177}$ L. Perniè, ${ }^{177}$ D. Rathjens, ${ }^{177}$ A. Safonov, ${ }^{177}$ N. Akchurin, ${ }^{178}$ J. Damgov, ${ }^{178}$ F. De Guio, ${ }^{178}$ P. R. Dudero, ${ }^{178}$ S. Kunori, ${ }^{178}$ K. Lamichhane, ${ }^{178}$ S. W. Lee, ${ }^{178}$ T. Mengke, ${ }^{178}$

S. Muthumuni, ${ }^{178}$ T. Peltola, ${ }^{178}$ S. Undleeb, ${ }^{178}$ I. Volobouev, ${ }^{178}$ Z. Wang, ${ }^{178}$ S. Greene, ${ }^{179}$ A. Gurrola, ${ }^{179}$ R. Janjam, ${ }^{179}$ W. Johns, ${ }^{179}$ C. Maguire, ${ }^{179}$ A. Melo, ${ }^{179}$ H. Ni,${ }^{179}$ K. Padeken, ${ }^{179}$ F. Romeo, ${ }^{179}$ J. D. Ruiz Alvarez, ${ }^{179}$ P. Sheldon, ${ }^{179}$ S. Tuo, ${ }^{179}$ J. Velkovska, ${ }^{179}$ M. Verweij, ${ }^{179}$ Q. Xu, ${ }^{179}$ M. W. Arenton, ${ }^{180}$ P. Barria, ${ }^{180}$ B. Cox,${ }^{180}$ R. Hirosky, ${ }^{180}$ M. Joyce, ${ }^{180}$ A. Ledovskoy, ${ }^{180}$ H. Li,${ }^{180}$ C. Neu, ${ }^{180}$ T. Sinthuprasith, ${ }^{180}$ Y. Wang, ${ }^{180}$ E. Wolfe, ${ }^{180}$ F. Xia,${ }^{180}$ R. Harr ${ }^{181}$ P. E. Karchin, ${ }^{181}$ N. Poudyal, ${ }^{181}$ J. Sturdy, ${ }^{181}$ P. Thapa ${ }^{181}$ S. Zaleski, ${ }^{181}$ J. Buchanan, ${ }^{182}$ C. Caillol, ${ }^{182}$ D. Carlsmith, ${ }^{182}$ S. Dasu, ${ }^{182}$ I. De Bruyn, ${ }^{182}$ L. Dodd, ${ }^{182}$ B. Gomber, ${ }^{182}$ M. Grothe, ${ }^{182}$ M. Herndon, ${ }^{182}$ A. Hervé, ${ }^{182}$ U. Hussain, ${ }^{182}$ P. Klabbers, ${ }^{182}$ A. Lanaro, ${ }^{182}$ K. Long, ${ }^{182}$ R. Loveless, ${ }^{182}$ T. Ruggles, ${ }^{182}$ A. Savin, ${ }^{182}$ V. Sharma, ${ }^{182}$ N. Smith, ${ }^{182}$ W. H. Smith, ${ }^{182}$ and N. Woods ${ }^{182}$

(CMS Collaboration)

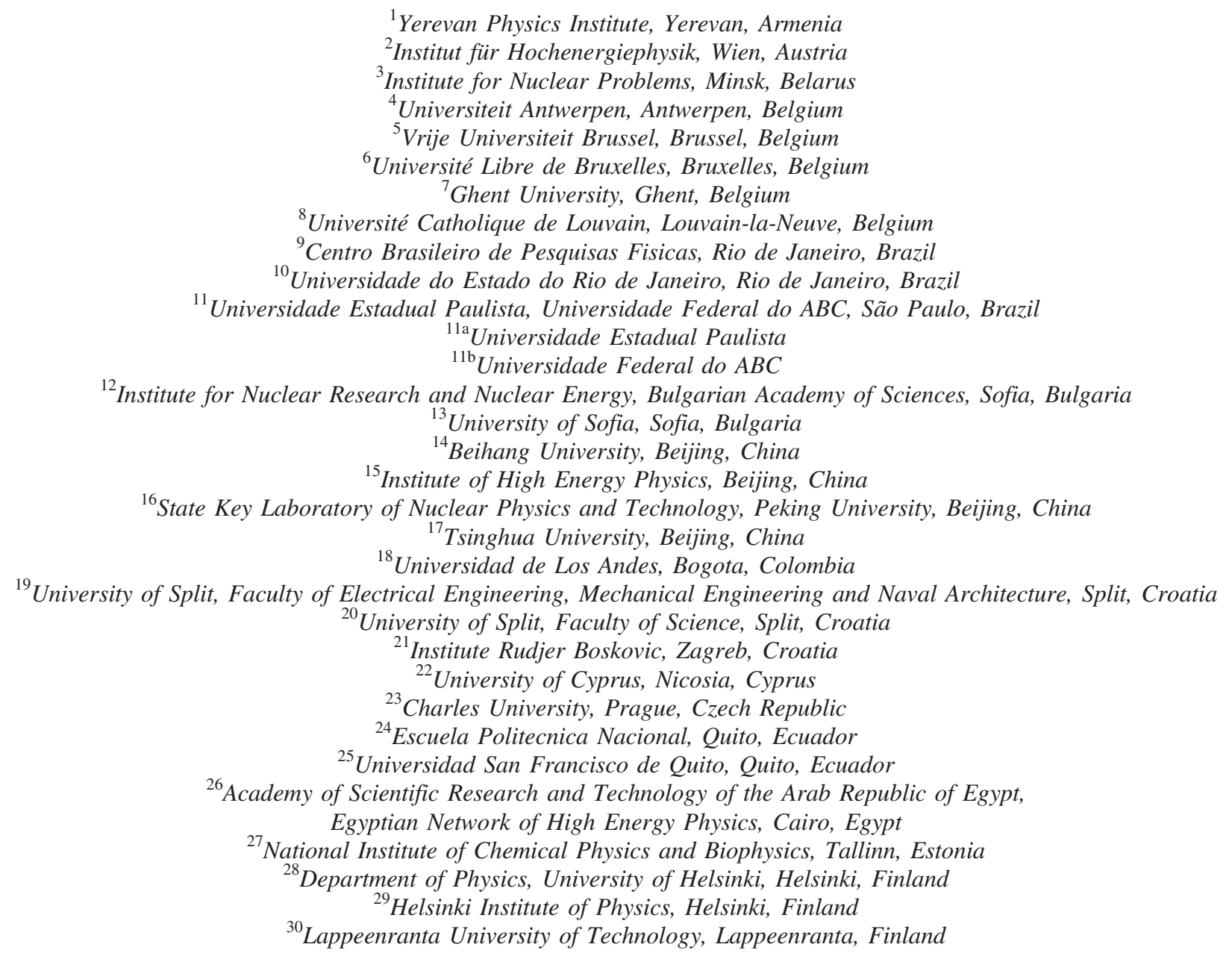


${ }^{31} I R F U, C E A$, Université Paris-Saclay, Gif-sur-Yvette, France

${ }^{32}$ Laboratoire Leprince-Ringuet, Ecole polytechnique, CNRS/IN2P3, Université Paris-Saclay, Palaiseau, France

${ }^{33}$ Université de Strasbourg, CNRS, IPHC UMR 7178, Strasbourg, France

${ }^{34}$ Centre de Calcul de l'Institut National de Physique Nucleaire et de Physique des Particules, CNRS/IN2P3, Villeurbanne, France

${ }^{35}$ Université de Lyon, Université Claude Bernard Lyon 1, CNRS-IN2P3, Institut de Physique Nucléaire de Lyon, Villeurbanne, France

${ }^{36}$ Georgian Technical University, Tbilisi, Georgia

${ }^{37}$ Tbilisi State University, Tbilisi, Georgia

${ }^{38}$ RWTH Aachen University, I. Physikalisches Institut, Aachen, Germany

${ }^{39}$ RWTH Aachen University, III. Physikalisches Institut A, Aachen, Germany

${ }^{40}$ RWTH Aachen University, III. Physikalisches Institut B, Aachen, Germany

${ }^{41}$ Deutsches Elektronen-Synchrotron, Hamburg, Germany

${ }^{42}$ University of Hamburg, Hamburg, Germany

${ }^{43}$ Karlsruher Institut fuer Technologie, Karlsruhe, Germany

${ }^{44}$ Institute of Nuclear and Particle Physics (INPP), NCSR Demokritos, Aghia Paraskevi, Greece

${ }^{45}$ National and Kapodistrian University of Athens, Athens, Greece

${ }^{46}$ National Technical University of Athens, Athens, Greece

${ }^{47}$ University of Ioánnina, Ioánnina, Greece

${ }^{48}$ MTA-ELTE Lendület CMS Particle and Nuclear Physics Group, Eötvös Loránd University, Budapest, Hungary

${ }^{49}$ Wigner Research Centre for Physics, Budapest, Hungary

${ }^{50}$ Institute of Nuclear Research ATOMKI, Debrecen, Hungary

${ }^{51}$ Institute of Physics, University of Debrecen, Debrecen, Hungary

${ }^{52}$ Indian Institute of Science (IISc), Bangalore, India

${ }^{53}$ National Institute of Science Education and Research, HBNI, Bhubaneswar, India

${ }^{54}$ Panjab University, Chandigarh, India

${ }^{55}$ University of Delhi, Delhi, India

${ }^{56}$ Saha Institute of Nuclear Physics, HBNI, Kolkata,India

${ }^{57}$ Indian Institute of Technology Madras, Madras, India

${ }^{58}$ Bhabha Atomic Research Centre, Mumbai, India

${ }^{59}$ Tata Institute of Fundamental Research-A, Mumbai, India

${ }^{60}$ Tata Institute of Fundamental Research-B, Mumbai, India

${ }^{61}$ Indian Institute of Science Education and Research (IISER), Pune, India

${ }^{62}$ Institute for Research in Fundamental Sciences (IPM), Tehran, Iran

${ }^{63}$ University College Dublin, Dublin, Ireland

${ }^{64}$ INFN Sezione di Bari, Università di Bari, Politecnico di Bari, Bari, Italy

${ }^{64 a}$ INFN Sezione di Bari, Bari, Italy

${ }^{64 \mathrm{~b}}$ Università di Bari, Bari, Italy

${ }^{64 \mathrm{c}}$ Politecnico di Bari, Bari, Italy

${ }^{65}$ INFN Sezione di Bologna, Università di Bologna, Bologna, Italy

${ }^{65 a}$ INFN Sezione di Bologna, Bologna, Italy

${ }^{65 \mathrm{~b}}$ Università di Bologna, Bologna, Italy

${ }^{66}$ INFN Sezione di Catania, Università di Catania, Catania, Italy

${ }^{66 a}$ INFN Sezione di Catania, Catania, Italy

${ }^{66 \mathrm{~b}}$ Università di Catania, Catania, Italy

${ }^{67}$ INFN Sezione di Firenze, Università di Firenze, Firenze, Italy

${ }^{67 \mathrm{a}}$ INFN Sezione di Firenze, Firenze, Italy

${ }^{67 \mathrm{~b}}$ Università di Firenze, Firenze, Italy

${ }^{68}$ INFN Laboratori Nazionali di Frascati, Frascati, Italy

${ }^{69}$ INFN Sezione di Genova, Università di Genova, Genova, Italy

${ }^{69 a}$ INFN Sezione di Genova, Genova, Italy

${ }^{69 \mathrm{~b}}$ Università di Genova, Genova, Italy

${ }^{70}$ INFN Sezione di Milano-Bicocca, Università di Milano-Bicocca, Milano, Italy

${ }^{70 a}$ INFN Sezione di Milano-Bicocca, Milano, Italy

${ }^{70 \mathrm{~b}}$ Università di Milano-Bicocca, Milano, Italy

${ }^{71}$ INFN Sezione di Napoli, Università di Napoli 'Federico II', Napoli, Italy, Università della Basilicata,

Potenza, Italy, Università G. Marconi, Roma, Italy

${ }^{71}$ INFN Sezione di Napoli, Napoli, Italy

${ }^{71 \mathrm{~b}}$ Università di Napoli 'Federico II', Napoli, Italy

${ }^{71 \mathrm{c}}$ Università della Basilicata, Potenza, Italy

${ }^{71 \mathrm{~d}}$ Università G. Marconi, Roma, Italy

${ }^{72}$ INFN Sezione di Padova, Università di Padova, Padova, Italy, Università di Trento, Trento, Italy 
${ }^{72 a}$ INFN Sezione di Padova, Padova, Italy

${ }^{72 \mathrm{~b}}$ Università di Padova, Padova, Italy

${ }^{72 \mathrm{c}}$ Università di Trento, Trento, Italy

${ }^{73 a}$ INFN Sezione di Pavia, Pavia, Italy

${ }^{73 b}$ Università di Pavia, Pavia, Italy

${ }^{74}$ INFN Sezione di Perugia, Università di Perugia, Perugia, Italy

${ }^{74 a}$ INFN Sezione di Perugia, Perugia, Italy

${ }^{74 \mathrm{~b}}$ Università di Perugia, Perugia, Italy

${ }^{75}$ INFN Sezione di Pisa, Università di Pisa, Scuola Normale Superiore di Pisa, Pisa, Italy

${ }^{75 a}$ INFN Sezione di Pisa, Pisa, Italy

${ }^{75 b}$ Università di Pisa, Pisa, Italy

${ }^{75 c}$ Scuola Normale Superiore di Pisa, Pisa, Italy

${ }^{76}$ INFN Sezione di Roma, Sapienza Università di Roma, Rome, Italy

${ }^{76 a}$ INFN Sezione di Roma, Rome, Italy

${ }^{76 \mathrm{~b}}$ Sapienza Università di Roma, Rome, Italy

${ }^{77}$ INFN Sezione di Torino, Università di Torino, Torino, Italy, Università del Piemonte Orientale, Novara, Italy

${ }^{77 a}$ INFN Sezione di Torino, Torino, Italy

${ }^{77 b}$ Università di Torino, Torino, Italy

${ }^{77 \mathrm{c}}$ Università del Piemonte Orientale, Novara, Italy

${ }^{78}$ INFN Sezione di Trieste, Università di Trieste, Trieste, Italy

${ }^{78 a}$ INFN Sezione di Trieste, Trieste, Italy

${ }^{78 \mathrm{~b}}$ Università di Trieste, Trieste, Italy

${ }^{79}$ Kyungpook National University, Daegu, Korea

${ }^{80}$ Chonnam National University, Institute for Universe and Elementary Particles, Kwangju, Korea

${ }^{81}$ Hanyang University, Seoul, Korea

${ }^{82}$ Korea University, Seoul, Korea

${ }^{83}$ Sejong University, Seoul, Korea

${ }^{84}$ Seoul National University, Seoul, Korea

${ }^{85}$ University of Seoul, Seoul, Korea

${ }^{86}$ Sungkyunkwan University, Suwon, Korea

${ }^{87}$ Vilnius University, Vilnius, Lithuania

${ }^{88}$ National Centre for Particle Physics, Universiti Malaya, Kuala Lumpur, Malaysia

${ }^{89}$ Universidad de Sonora (UNISON), Hermosillo, Mexico

${ }^{90}$ Centro de Investigacion y de Estudios Avanzados del IPN, Mexico City, Mexico

${ }^{91}$ Universidad Iberoamericana, Mexico City, Mexico

${ }^{92}$ Benemerita Universidad Autonoma de Puebla, Puebla, Mexico

${ }^{93}$ Universidad Autónoma de San Luis Potosí, San Luis Potosí, Mexico

${ }^{94}$ University of Auckland, Auckland, New Zealand

${ }^{95}$ University of Canterbury, Christchurch, New Zealand

${ }^{96}$ National Centre for Physics, Quaid-I-Azam University, Islamabad, Pakistan

${ }^{97}$ National Centre for Nuclear Research, Swierk, Poland

${ }^{98}$ Institute of Experimental Physics, Faculty of Physics, University of Warsaw, Warsaw, Poland

${ }^{99}$ Laboratório de Instrumentação e Física Experimental de Partículas, Lisboa, Portugal

${ }^{100}$ Joint Institute for Nuclear Research, Dubna, Russia

${ }^{101}$ Petersburg Nuclear Physics Institute, Gatchina (St. Petersburg), Russia

${ }^{102}$ Institute for Nuclear Research, Moscow, Russia

${ }^{103}$ Institute for Theoretical and Experimental Physics, Moscow, Russia

${ }^{104}$ Moscow Institute of Physics and Technology, Moscow, Russia

${ }^{105}$ National Research Nuclear University 'Moscow Engineering Physics Institute' (MEPhI), Moscow, Russia

${ }^{106}$ P.N. Lebedev Physical Institute, Moscow, Russia

${ }^{107}$ Skobeltsyn Institute of Nuclear Physics, Lomonosov Moscow State University, Moscow, Russia

${ }^{108}$ Novosibirsk State University (NSU), Novosibirsk, Russia

${ }^{109}$ Institute for High Energy Physics of National Research Centre 'Kurchatov Institute', Protvino, Russia

${ }^{110}$ National Research Tomsk Polytechnic University, Tomsk, Russia

${ }^{111}$ University of Belgrade, Faculty of Physics and Vinca Institute of Nuclear Sciences, Belgrade, Serbia

${ }^{112}$ Centro de Investigaciones Energéticas Medioambientales y Tecnológicas (CIEMAT), Madrid, Spain

${ }^{113}$ Universidad Autónoma de Madrid, Madrid, Spain

${ }^{114}$ Universidad de Oviedo, Oviedo, Spain

${ }^{115}$ Instituto de Física de Cantabria (IFCA), CSIC-Universidad de Cantabria, Santander, Spain

${ }^{116}$ University of Ruhuna, Department of Physics, Matara, Sri Lanka 


\footnotetext{
${ }^{117}$ CERN, European Organization for Nuclear Research, Geneva, Switzerland

${ }^{118}$ Paul Scherrer Institut, Villigen, Switzerland

${ }^{119}$ ETH Zurich-Institute for Particle Physics and Astrophysics (IPA), Zurich, Switzerland

${ }^{120}$ Universität Zürich, Zurich, Switzerland

${ }^{121}$ National Central University, Chung-Li, Taiwan

${ }^{122}$ National Taiwan University (NTU), Taipei, Taiwan

${ }^{123}$ Chulalongkorn University, Faculty of Science, Department of Physics, Bangkok, Thailand

${ }^{124}$ Cukurova University, Physics Department, Science and Art Faculty, Adana, Turkey

${ }^{125}$ Middle East Technical University, Physics Department, Ankara, Turkey

${ }^{126}$ Bogazici University, Istanbul, Turkey

${ }^{127}$ Istanbul Technical University, Istanbul, Turkey

${ }^{128}$ Institute for Scintillation Materials of National Academy of Science of Ukraine, Kharkov, Ukraine

${ }^{129}$ National Scientific Center, Kharkov Institute of Physics and Technology, Kharkov, Ukraine

${ }^{130}$ University of Bristol, Bristol, United Kingdom

${ }^{131}$ Rutherford Appleton Laboratory, Didcot, United Kingdom

${ }^{132}$ Imperial College, London, United Kingdom

${ }^{133}$ Brunel University, Uxbridge, United Kingdom

${ }^{134}$ Baylor University, Waco, Texas, USA

${ }^{135}$ Catholic University of America, Washington DC, USA

${ }^{136}$ The University of Alabama, Tuscaloosa, USA

${ }^{137}$ Boston University, Boston, Massachusetts, USA

${ }^{138}$ Brown University, Providence, Rhode Island, USA

${ }^{139}$ University of California, Davis, Davis, California, USA

${ }^{140}$ University of California, Los Angeles, California, USA

${ }^{141}$ University of California, Riverside, Riverside, California, USA

${ }^{142}$ University of California, San Diego, La Jolla, California, USA

${ }^{143}$ University of California, Santa Barbara-Department of Physics, Santa Barbara, California, USA

${ }^{144}$ California Institute of Technology, Pasadena, California, USA

${ }^{145}$ Carnegie Mellon University, Pittsburgh, Pennsylvania, USA

${ }^{146}$ University of Colorado Boulder, Boulder, Colorado, USA

${ }^{147}$ Cornell University, Ithaca, New York, USA

${ }^{148}$ Fermi National Accelerator Laboratory, Batavia, Illinois, USA

${ }^{149}$ University of Florida, Gainesville, Florida, USA

${ }^{150}$ Florida International University, Miami, Florida, USA

${ }^{151}$ Florida State University, Tallahassee, Florida, USA

${ }^{152}$ Florida Institute of Technology, Melbourne, Florida, USA

${ }^{153}$ University of Illinois at Chicago (UIC), Chicago, Illinois, USA

${ }^{154}$ The University of Iowa, Iowa City, Iowa, USA

${ }^{155}$ Johns Hopkins University, Baltimore, Maryland, USA

${ }^{156}$ The University of Kansas, Lawrence, Kansas, USA

${ }^{157}$ Kansas State University, Manhattan, Kansas, USA

${ }^{158}$ Lawrence Livermore National Laboratory, Livermore, California, USA

${ }^{159}$ University of Maryland, College Park, Maryland, USA

${ }^{160}$ Massachusetts Institute of Technology, Cambridge, Massachusetts, USA

${ }^{161}$ University of Minnesota, Minneapolis, Minnesota, USA

${ }^{162}$ University of Mississippi, Oxford, Mississippi, USA

${ }^{163}$ University of Nebraska-Lincoln, Lincoln, Nebraska, USA

${ }^{164}$ State University of New York at Buffalo, Buffalo, New York, USA

${ }^{165}$ Northeastern University, Boston, Massachusetts, USA

${ }^{166}$ Northwestern University, Evanston, Illinois, USA

${ }^{167}$ University of Notre Dame, Notre Dame, Indiana, USA

${ }^{168}$ The Ohio State University, Columbus, Ohio, USA

${ }^{169}$ Princeton University, Princeton, New Jersey, USA

${ }^{170}$ University of Puerto Rico, Mayaguez, Puerto Rico, USA

${ }^{171}$ Purdue University, West Lafayette, Indiana, USA

${ }^{172}$ Purdue University Northwest, Hammond, Indiana, USA

${ }^{173}$ Rice University, Houston, Texas, USA

${ }^{174}$ University of Rochester, Rochester, New York, USA

${ }^{175}$ Rutgers, The State University of New Jersey, Piscataway, New Jersey, USA

${ }^{176}$ University of Tennessee, Knoxville, Tennessee, USA
} 


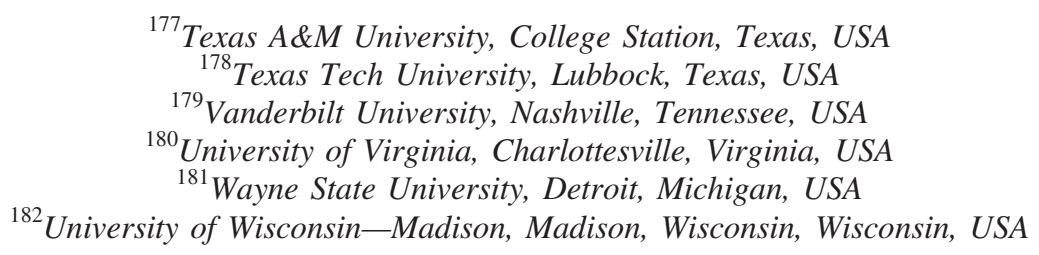

${ }^{\mathrm{a}}$ Deceased.

${ }^{\mathrm{b}}$ Also at Vienna University of Technology, Vienna, Austria.

${ }^{c}$ Also at IRFU, CEA, Université Paris-Saclay, Gif-sur-Yvette, France.

${ }^{\mathrm{d}}$ Also at Universidade Estadual de Campinas, Campinas, Brazil.

${ }^{\mathrm{e}}$ Also at Federal University of Rio Grande do Sul, Porto Alegre, Brazil.

${ }^{\mathrm{f}}$ Also at Université Libre de Bruxelles, Bruxelles, Belgium.

${ }^{\mathrm{g}}$ Also at University of Chinese Academy of Sciences.

${ }^{\mathrm{h}}$ Also at Institute for Theoretical and Experimental Physics, Moscow, Russia.

${ }^{\mathrm{i}}$ Also at Joint Institute for Nuclear Research, Dubna, Russia.

${ }^{\mathrm{j}}$ Also at Cairo University, Cairo, Egypt.

${ }^{\mathrm{k}}$ Also at Helwan University, Cairo, Egypt.

${ }^{1}$ Also at Zewail City of Science and Technology, Zewail, Egypt.

${ }^{\mathrm{m}}$ Also at British University in Egypt, Cairo, Egypt.

${ }^{\mathrm{n}}$ Also at Fayoum University, El-Fayoum, Egypt.

${ }^{\circ}$ Also at Department of Physics, King Abdulaziz University, Jeddah, Saudi Arabia.

${ }^{\mathrm{p}}$ Also at Université de Haute Alsace, Mulhouse, France.

${ }^{\mathrm{q}}$ Also at Skobeltsyn Institute of Nuclear Physics, Lomonosov Moscow State University, Moscow, Russia.

${ }^{\mathrm{r}}$ Also at CERN, European Organization for Nuclear Research, Geneva, Switzerland.

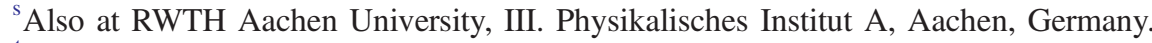

${ }^{\mathrm{t}}$ Also at University of Hamburg, Hamburg, Germany.

"Also at Brandenburg University of Technology, Cottbus, Germany.

${ }^{\mathrm{v}}$ Also at Institute of Physics, University of Debrecen, Debrecen, Hungary.

${ }^{\text {w }}$ Also at Institute of Nuclear Research ATOMKI, Debrecen, Hungary.

${ }^{x}$ Also at MTA-ELTE Lendület CMS Particle and Nuclear Physics Group, Eötvös Loránd University, Budapest, Hungary.

${ }^{\mathrm{y}}$ Also at IIT Bhubaneswar, Bhubaneswar, India.

${ }^{\mathrm{z}}$ Also at Institute of Physics, Bhubaneswar, India.

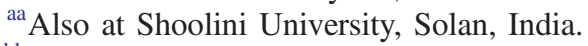

${ }^{\mathrm{bb}}$ Also at University of Visva-Bharati, Santiniketan, India.

${ }^{c c}$ Also at Isfahan University of Technology, Isfahan, Iran.

${ }^{\mathrm{dd}}$ Also at Plasma Physics Research Center, Science and Research Branch, Islamic Azad University, Tehran, Iran.

${ }^{\mathrm{ee}}$ Also at Italian National Agency for New Technologies, Energy and Sustainable Economic Development, Bologna, Italy.

${ }^{\mathrm{ff}}$ Also at Università degli Studi di Siena, Siena, Italy.

${ }^{\mathrm{gg}}$ Also at Scuola Normale e Sezione dell'INFN, Pisa, Italy.

${ }^{\text {hh }}$ Also at Kyunghee University, Seoul, Korea.

${ }^{\text {ii }}$ Also at International Islamic University of Malaysia, Kuala Lumpur, Malaysia.

${ }^{\mathrm{jj}}$ Also at Malaysian Nuclear Agency, MOSTI, Kajang, Malaysia.

${ }^{k k}$ Also at Consejo Nacional de Ciencia y Tecnología, Mexico city, Mexico.

${ }^{11}$ Also at Warsaw University of Technology, Institute of Electronic Systems, Warsaw, Poland.

${ }^{\mathrm{mm}}$ Also at Institute for Nuclear Research, Moscow, Russia.

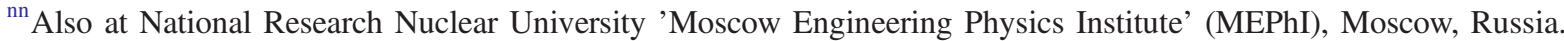

${ }^{\text {oo }}$ Also at St. Petersburg State Polytechnical University, St. Petersburg, Russia.

${ }^{\mathrm{pp}}$ Also at University of Florida, Gainesville, Florida, USA.

${ }^{\mathrm{qq}}$ Also at P.N. Lebedev Physical Institute, Moscow, Russia.

${ }^{\mathrm{rr}}$ Also at California Institute of Technology, Pasadena, California, USA.

${ }^{\text {ss }}$ Also at Budker Institute of Nuclear Physics, Novosibirsk, Russia.

${ }^{t t}$ Also at Faculty of Physics, University of Belgrade, Belgrade, Serbia.

${ }^{u u}$ Also at INFN Sezione di Pavia, Università di Pavia, Pavia, Italy.

${ }^{\mathrm{vv}}$ Also at University of Belgrade, Faculty of Physics and Vinca Institute of Nuclear Sciences, Belgrade, Serbia.

${ }^{w w}$ Also at National and Kapodistrian University of Athens, Athens, Greece.

${ }^{\mathrm{xx}}$ Also at Riga Technical University, Riga, Latvia.

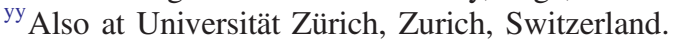

${ }^{\mathrm{zz}}$ Also at Stefan Meyer Institute for Subatomic Physics, Vienna, Austria.

${ }^{\text {aaa } A l s o ~ a t ~ A d i y a m a n ~ U n i v e r s i t y, ~ A d i y a m a n, ~ T u r k e y . ~}$ 


\footnotetext{
${ }^{\text {bbb }}$ Also at Istanbul Aydin University, Istanbul, Turkey.

${ }^{c c c}$ Also at Mersin University, Mersin, Turkey.

${ }^{\text {ddd }}$ Also at Piri Reis University, Istanbul, Turkey.

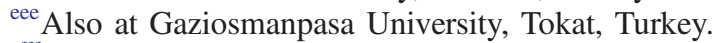

${ }^{\mathrm{fff}}$ Also at Ozyegin University, Istanbul, Turkey.

${ }^{\text {ggg }}$ Also at Izmir Institute of Technology, Izmir, Turkey.

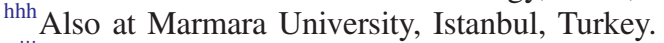

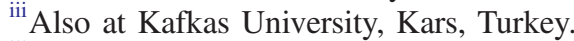

${ }^{\mathrm{jjj}}$ Also at Istanbul University, Faculty of Science, Istanbul, Turkey.

${ }^{k k k}$ Also at Istanbul Bilgi University, Istanbul, Turkey.

${ }^{11 l}$ Also at Hacettepe University, Ankara, Turkey.

${ }^{\mathrm{mmm}}$ Also at Rutherford Appleton Laboratory, Didcot, United Kingdom.

${ }^{n n n}$ Also at School of Physics and Astronomy, University of Southampton, Southampton, United Kingdom.

${ }^{000}$ Also at Monash University, Faculty of Science, Clayton, Australia.

${ }^{\mathrm{ppp}}$ Also at Bethel University, St. Paul, USA.

${ }^{\mathrm{qqq}}$ Also at Karamanoğlu Mehmetbey University, Karaman, Turkey.

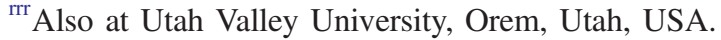

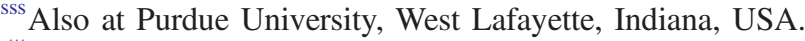

${ }^{t t t}$ Also at Beykent University, Istanbul, Turkey.

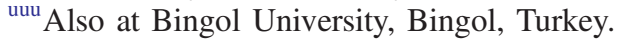

${ }^{\mathrm{vvv}}$ Also at Sinop University, Sinop, Turkey.

${ }^{w w w}$ Also at Mimar Sinan University, Istanbul, Istanbul, Turkey.

${ }^{\mathrm{xxx}}$ Also at Texas A\&M University at Qatar, Doha, Qatar.

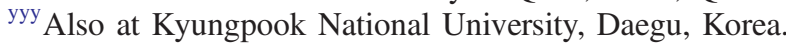

